\title{
4. PLATINUM-GROUP-ELEMENT CONCENTRATIONS IN MAFIC AND ULTRAMAFIC LITHOLOGIES DRILLED FROM HESS DEEP ${ }^{1}$
}

\author{
H.M. Prichard, ${ }^{2}$ H. Puchelt,${ }^{3}$ J.-D. Eckhardt, ${ }^{3}$ and P.C. Fisher ${ }^{2}$
}

\begin{abstract}
This paper reports the first concentrations of platinum-group minerals (PGM) and platinum-group elements (PGE), of tens of parts per billion, to be described from the lower oceanic crust exposed in Hess Deep, if not the first concentrations reported from modern-day unsubducted lower oceanic crust. The results indicate that detectable PGE concentrations occur in fastspreading oceanic crust. The PGE-bearing melt extracted from the mantle provides the potential for PGE to be concentrated in lower oceanic crustal ultramafic rocks, and this study has identified such concentrations in the magmatic plutonic lithologies from Hess Deep. However, PGE concentrations calculated in $100 \%$ of sulfide reveal that sulfides in the residual mantle harzburgite at Hess Deep are enriched in PGE relative to lower crustal dunite. Therefore, the harzburgites retain some PGE and remain fertile for the extraction of more PGE during subduction.

Of the high-level gabbros from Site 894 and gabbro, troctolite, dunite, and harzburgite from Site 895, the troctolite from Holes $895 \mathrm{D}$ and $895 \mathrm{E}$ was found to have the maximum values of $\mathrm{Pt}(36 \mathrm{ppb})$ and $\mathrm{Pd}(54 \mathrm{ppb})$, together with a dunite from Hole $895 \mathrm{E}$, which had maximum values of $\mathrm{Pt}(24 \mathrm{ppb})$ and $\mathrm{Pd}(46 \mathrm{ppb})$. In both lithologies, the greatest values of PGE coincide with the greatest modal abundance of base-metal sulfide and chrome-spinel. Detectable values of PGE were observed also in gabbro and harzburgite and the slightly more PGE-enriched gabbros are also those with relatively depleted concentrations of rare earth elements (REE).

PGM have been located in both dunite and troctolite. In the troctolite, the most common PGM are alloys of precious and base metals, specifically Pd-Sn-Cu, Cu-Pt-Au, Pt-Fe-Ni-Cu, and Ni-Pt-Fe. One Pd-Bi-Te mineral was observed. These PGM are associated with chrome-spinel and pentlandite, which is altering to magnetite, millerite, $\mathrm{Ni}-\mathrm{Fe}$ alloys, and oxides. Cu-bearing sulfides and native $\mathrm{Cu}$ are present. In the dunite, an Ir-Pt alloy and a Pd-Sn alloy occur close together within a Ni-Fe alloy.
\end{abstract}

\section{INTRODUCTION}

Ocean Drilling Program (ODP) Leg 147 drilled six short holes into transition zone mafic and ultramafic lithologies exposed in the Hess Deep fracture zone at $2^{\circ} \mathrm{N}, 101^{\circ} \mathrm{W}$. Cores from Holes $895 \mathrm{C}$, $895 \mathrm{D}$, and $895 \mathrm{E}$ contain sequences of dunite, harzburgite, troctolite, and gabbro and are some of the first sections of deep-level oceanic crust to be recovered from the Pacific Ocean. These samples provide a unique opportunity to examine the concentrations of platinumgroup elements (PGE) in unsubducted lower oceanic crust formed at a fast-spreading ridge. High-level gabbro from Site 894was also sampled for PGE analysis. The aim of this research was to (1) identify PGE concentrations in the different lithologies within the drill cores, (2) locate platinum-group minerals (PGM), and so (3) examine the processes of extraction of PGE from the mantle and the elements' reconcentration in the oceanic crust. Also, access to this Hess Deep core permits a comparison of PGE concentrations in the very young unsubducted oceanic crust, formed by fast spreading, with ancient oceanic crust exposed in ophiolite complexes.

\section{Sampling}

$\mathrm{Pt}$ and $\mathrm{Pd}$ are known to be chalcophile and, consequently, are commonly associated with sulfide minerals in natural rock systems

'Mével, C., Gillis, K.M., Allan, J.F., and Meyer, P.S. (Eds.), 1996. Proc. ODP, Sci. Results, 147: College Station, TX (Ocean Drilling Program).

'Geology Department, University of Wales College of Cardiff, PO Box 914, Cardiff, CF1 3YE, Wales, United Kingdom. sglhmp@cardiff.ac.uk

'Institut für Petrographie und Geochemie der Universität Karlsruhe, Kaiserstrasse 12, D-7500 Karlsruhe, Federal Republic of Germany,dg09@rz.uni-karlsruhe.de
(Naldrett, 1989). In contrast, Os, Ir, and Ru are concentrated with chromite (Von Gruenewaldt et al., 1986). Research for PGE concentrations in ophiolite complexes has revealed the presence of significant concentrations of $\mathrm{Os}, \mathrm{Ir}$, and $\mathrm{Ru}$ with chromite (Agioritis and Wolf, 1978; Page et al., 1982, 1984; Page and Talkington, 1984) and more recently Pt and Pd with sulfides (Prichard et al., 1986; Ohnenstetter et al., 1991; Prichard and Lord, 1993; Pedersen et al., 1993). With this knowledge, the sampling program for this study was designed to test whether PGE are concentrated with sulfides and/or chromite in the Hess Deep core. Each piece of core was examined aboard ship under a binocular microscope for the presence of sulfide and chromite, and the percentages of each were estimated and logged (Figs. 1,2). Core pieces with the highest modal percentage of visible sulfide and chromite were sampled. Barren samples were collected as controls with the expectation that these would be low in PGE.

\section{Analytical Techniques}

Conventionally, for PGE analysis, 1-kg samples are homogenized to avoid the nugget effect, in which PGE unevenly distributed in a sample can give rise to unrepresentative results if the sample size is too small. The samples homogenized in this study were approximately $50 \mathrm{~g}$, owing to the necessity for sampling restrictions due to the limited quantity of core material available. However, the results appear satisfactory, as it was possible to interpret the results within their geological context and PGM were located in samples with the greatest PGE values. Altogether, 54 samples were analyzed for the six PGE using a Ni-sulfide fire-assay preconcentration technique followed by an ICP-MS finish. Two independent sets of analyses (Tables 1,2 ) were obtained from two laboratories, one available to the British authors and the other to the German authors. Data set 1 (Table 1) consists of analyses of samples collected specifically to locate 

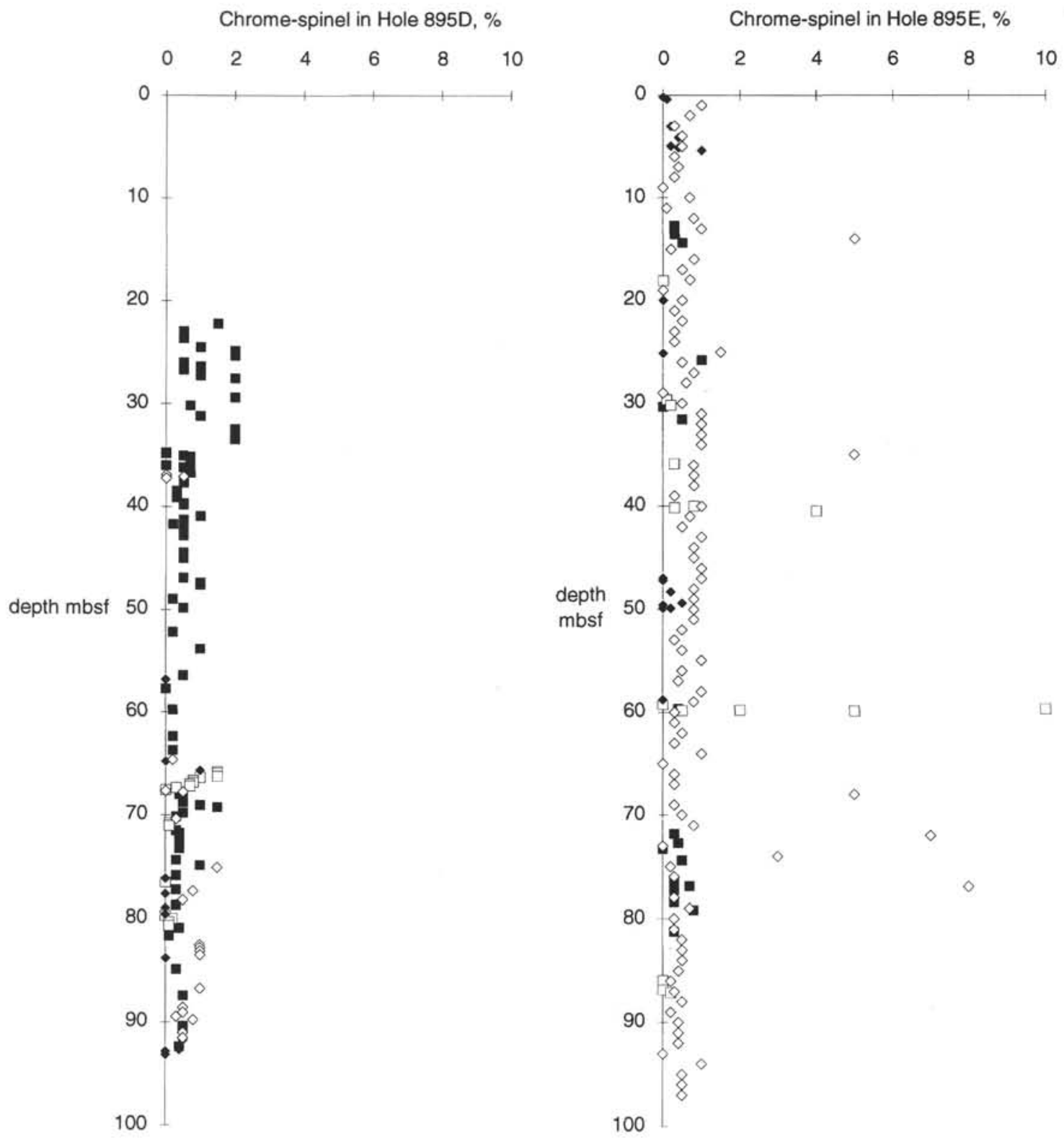

Figure 1. Modal proportions of chromite in core from Holes $895 \mathrm{D}$ and $895 \mathrm{E}$ as observed in hand specimen plotted against depth in meters below seafloor (mbsf). Each section of core recorded is logged by lithology, with harzburgites as solid squares, troctolites as open squares, gabbros as solid diamonds, and dunites as open diamonds.

maximum PGE concentrations within the cores whereas data set 2 (Table 2) is more comprehensive and consists of analyzes of samples collected to study the systematic average variations in PGE concentrations throughout all the lithologies recovered at Sites 894 and 895 and so contains more analyses of PGE-poor lithologies such as gabbro from Site 894 and harzburgite from Site 895 . The detection limits were approximately $2 \mathrm{ppb}$ for set 1 (Table 1 ) and approximately 1.5 $\mathrm{ppb}$ for $\mathrm{Pd}, 1 \mathrm{ppb}$ for $\mathrm{Ru}$ and $\mathrm{Rh}, 0.5 \mathrm{ppb}$ for Os, and $0.2 \mathrm{ppb}$ for Ir for set 2 (Table 2). Table 3 compares the values of PGE in three samples measured by both laboratories. Both laboratories reported maximum values for $\mathrm{Ru}, \mathrm{Pd}$, and $\mathrm{Pt}$ in troctolite Sample 147-895D-7R-1 (Piece 8, 45-49 cm). The values in set 1 are greater than in set 2 but the data may not be directly comparable because they were obtained from two adjacent small slices of Sample 147-895D-7R-1 (Piece 8, $45-49 \mathrm{~cm})$ and not from a homogenized powder from the whole piece. The two gabbro Samples 147-894G-11R-2 (Piece 14, 133-137 $\mathrm{cm}$ ) and 147-894G-13R-1 (Piece 7B, 42-48 cm) analyzed in duplicate give low but comparable values. Reference materials for low PGE concentrations are rare; a diluted form of the international standard SARM 7 was used for set 1 . For set 2, a reference material from a German cruise was used. This consisted of a core (95-somme 60 ) of sediment derived from ultramafic rocks, recovered at $2^{\circ} 14.29^{\prime} \mathrm{N}$, $101^{\circ} 32.88^{\prime} \mathrm{W}$. A set of repeated PGE analyses of the reference material is given in Table 4. The reference material was suitable for use as a standard for this study, with approximately similar PGE concentrations to the samples from Leg 147 of $3.6 \mathrm{ppb} \mathrm{Ru},<1 \mathrm{ppb} R \mathrm{Rh}, 4.5 \mathrm{ppb}$ $\mathrm{Pd},<0.5 \mathrm{ppb}$ Os, $1.7 \mathrm{ppb}$ Ir, and $3.4 \mathrm{ppb} \mathrm{Pt}$.

Pathfinder elements, which are typically associated with the precious metals, were analyzed by wavelength-dispersive X-ray fluorescence (XRF) for set 1 and energy-dispersive XRF and INAA for set 2 , using appropriate USGS reference powders and internal laboratory standards. The REE were measured by ICP-MS. The PGM were an- 
Sulfide in Hole 895D \%

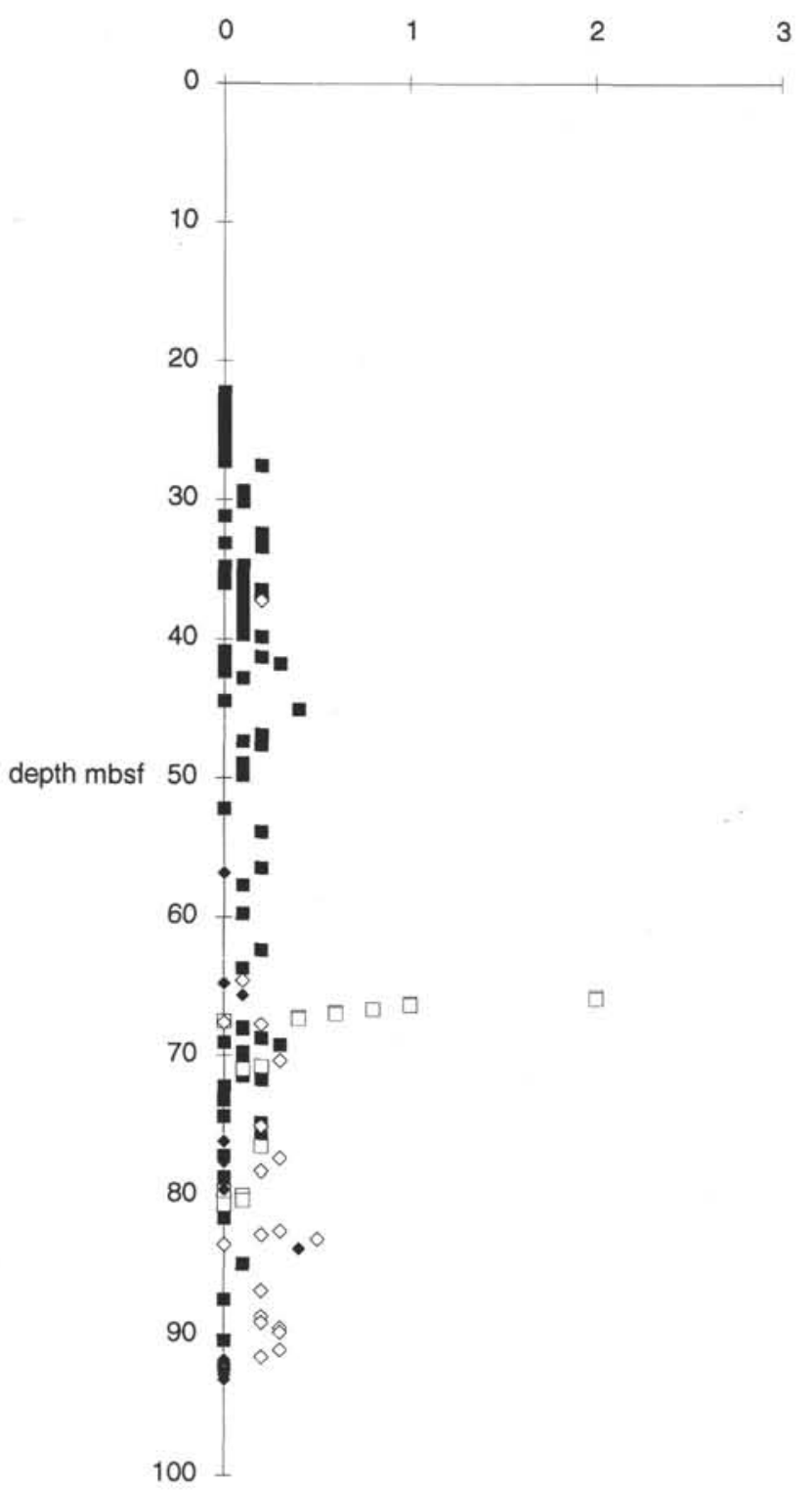

Sulfide in $895 \mathrm{E}, \%$

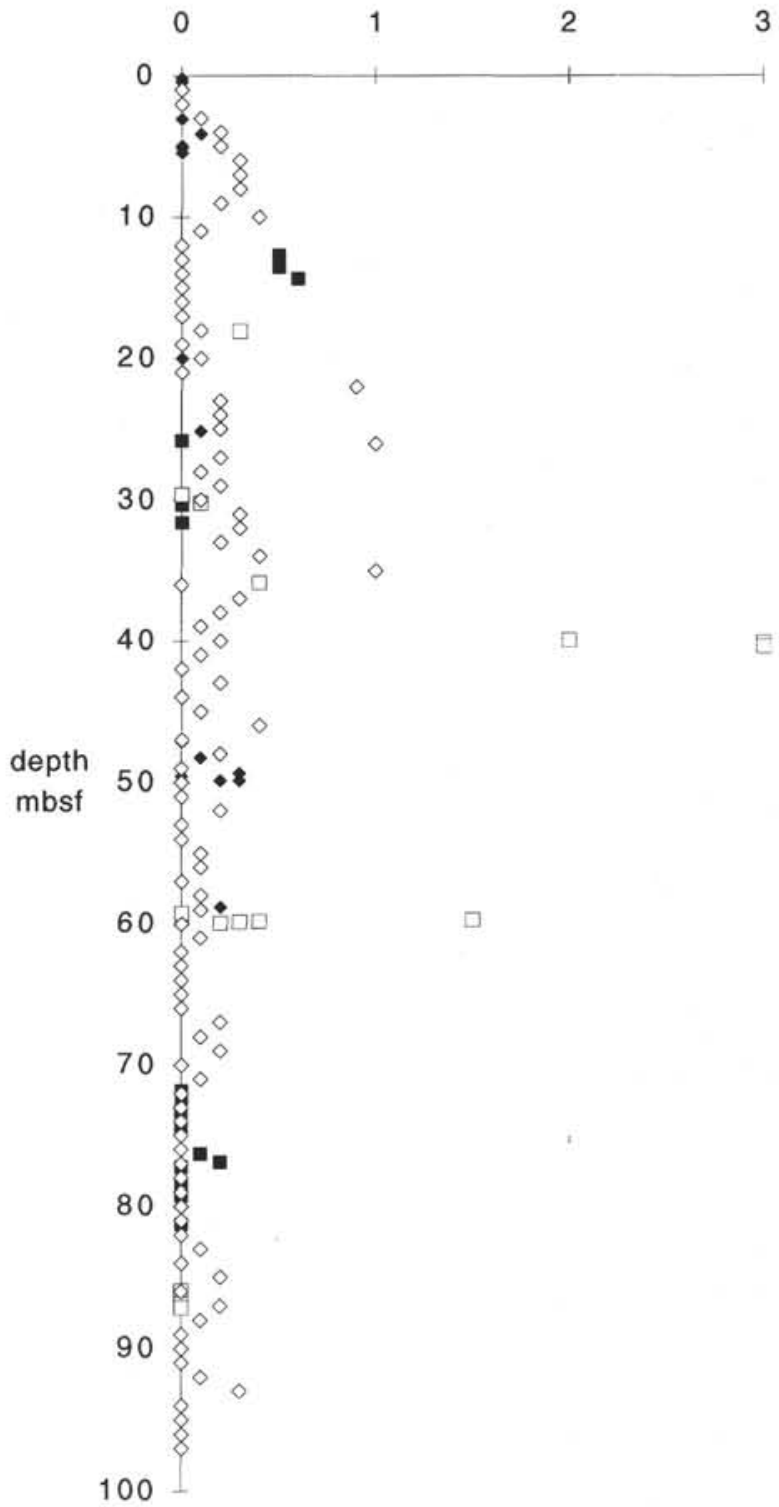

Figure 2. Modal proportions of sulfides in core from Holes $895 \mathrm{D}$ and $895 \mathrm{E}$ as observed in hand specimen plotted against depth. Each section of core recorded is logged by lithology, with symbols as in Figure 1.

alyzed using a Cambridge 360 scanning electron microscope with an energy-dispersive Link system with reference to a cobalt standard. The high resolution allowed analysis of PGM as small as $1 \mathrm{~mm}$ in their smallest diameter (Table 5). Some of the analyses of the smaller PGM contain $1 \%-2 \% \mathrm{Ni}$ and $\mathrm{Fe}$, which was most likely derived from the surrounding base-metal alloys where they are present in the same proportions as in these PGM (Table 5). For PGM too small to be analyzed quantitatively, elements were identified using energy-dispersive X-ray spectra (Fig. 3). The presence of oxygen in iron oxides was confirmed using a light-element EDS detector.

\section{RESULTS}

\section{Chromite and Sulfide Distribution}

Detailed observations of sulfides and chrome-spinel in the mafic/ ultramafic cores from Site 895 revealed specific concentrations of these minerals in certain lithologies (Figs. 1,2). Chromite is most abundant in dunites, as expected from a comparison of chromespinel concentrations in ophiolite complexes (Greenbaum, 1977; Neary and Brown, 1979; Roberts and Neary, 1993). The most abundant concentrations of chromite occur in Hole 895E, which has the greatest proportion of dunite. Thin chromite layers are developed especially at the base of the thickest dunite, for example, in Sample 147-895E-7R-1 (Piece 3). Unexpectedly, chrome-spinel is also systematically concentrated in the troctolites. In contrast, chrome-spinel is much less abundant in both the harzburgites and the gabbros. Sulfides were observed in all the lithologies at Site 895 but are systematically enriched in troctolite. In a troctolite interval from Section 147-895D-7R-1 (Piece 7) through (Piece 16), the sulfide content increases upward in association with a similar increase in chrome-spinel. In addition, sulfides are concentrated sporadically with chromite in the dunite and commonly were observed in the harzburgite. 
Table 1. PGE analyses for samples from Sites 894 and 895 , data set 1 .

\begin{tabular}{|c|c|c|c|c|c|c|c|c|c|c|c|c|c|}
\hline \multirow{2}{*}{$\begin{array}{l}\text { Core, section, } \\
\text { interval }(\mathrm{cm})\end{array}$} & \multirow[b]{2}{*}{ Lithology } & \multicolumn{6}{|c|}{ Platinum-group elements (ppb) } & \multicolumn{6}{|c|}{ Pathfinder elements (ppm) } \\
\hline & & Os & Ir & $\mathrm{Ru}$ & Rh & $\mathrm{Pt}$ & $\mathrm{Pd}$ & $\mathrm{Ni}$ & $\mathrm{Cu}$ & $\mathrm{Cr}$ & $\mathrm{Zn}$ & Co & V \\
\hline $\begin{array}{l}\text { 147-894E- } \\
\text { 3R-1 (Piecel, 0-4) }\end{array}$ & $\mathrm{g}$ & 0 & 2 & 4 & 1 & 6 & 8 & & & & & & \\
\hline $\begin{array}{l}\text { 147-894G- } \\
\text { 2R-1 (Piece 17, 127-134) } \\
\text { 2R-3 (Piece 1, 0-5) } \\
\text { 11R-2 (Piece 14, 133-137) } \\
\text { 13R-1 (Piece 7B, 42-48) } \\
\text { 13R-2 (Piece 9A, 100-107) }\end{array}$ & $\begin{array}{l}\text { BS } \\
\mathrm{g} \\
\mathrm{g} \\
\mathrm{g} \\
\mathrm{g}\end{array}$ & $\begin{array}{l}2 \\
2 \\
2 \\
2 \\
2\end{array}$ & $\begin{array}{l}2 \\
2 \\
2 \\
2 \\
2\end{array}$ & $\begin{array}{l}2 \\
2 \\
2 \\
0 \\
2\end{array}$ & $\begin{array}{l}1 \\
1 \\
1 \\
1 \\
1\end{array}$ & $\begin{array}{r}4 \\
6 \\
10 \\
4 \\
4\end{array}$ & $\begin{array}{l}2 \\
6 \\
4 \\
0 \\
0\end{array}$ & & & & & & \\
\hline $\begin{array}{l}\text { 147-845C- } \\
\text { 4R-1 (Piece 6C, 129-133) }\end{array}$ & tr & 2 & 2 & 6 & 1 & 10 & 14 & 1959 & 27 & 9889 & 63 & 127 & 81 \\
\hline $\begin{array}{l}\text { 147-895D- } \\
\text { 3R-1 (Piece 15, 133.0-139) } \\
\text { 7R-1 (Piece 8, 45-49) } \\
\text { 7R-1 (Piece 12, 74-79) } \\
\text { 7R-2 (Piece 2, 8.5-12) }\end{array}$ & $\begin{array}{l}\mathrm{hz} \\
\text { tr } \\
\text { tr } \\
\mathrm{hz}\end{array}$ & $\begin{array}{l}6 \\
4 \\
2 \\
6\end{array}$ & $\begin{array}{l}6 \\
4 \\
2 \\
6\end{array}$ & $\begin{array}{r}14 \\
8 \\
4 \\
12\end{array}$ & $\begin{array}{l}2 \\
3 \\
1 \\
2\end{array}$ & $\begin{array}{l}16 \\
36 \\
12 \\
16\end{array}$ & $\begin{array}{l}10 \\
44 \\
10 \\
14\end{array}$ & $\begin{array}{l}2261 \\
2368 \\
1811 \\
2253\end{array}$ & $\begin{array}{r}8 \\
318 \\
96 \\
7\end{array}$ & $\begin{array}{l}2497 \\
5980 \\
2330 \\
1736\end{array}$ & $\begin{array}{l}43 \\
48 \\
44 \\
45\end{array}$ & $\begin{array}{l}113 \\
115 \\
100 \\
118\end{array}$ & $\begin{array}{l}38 \\
42 \\
24 \\
25\end{array}$ \\
\hline $\begin{array}{l}\text { 147-895E- } \\
\text { 1R-3 (Piece 3B, 74-76) } \\
\text { 1R-4 (Piece 3, 28-32) } \\
\text { 4R-1 (Piece 19, 131-134) } \\
\text { 4R-2 (Piece 2C, 18-21) } \\
\text { 4R-2 (Piece 12, 105-109) } \\
\text { 6R-1 (Piece 17, 90-92) } \\
\text { 6R-4 (Piece 1D, 33-37) } \\
\text { 7R-1 (Piece 15B, 123-128) }\end{array}$ & $\begin{array}{l}d \\
d \\
d \\
d \\
d \\
\text { tr } \\
d \\
d\end{array}$ & $\begin{array}{l}6 \\
6 \\
6 \\
6 \\
6 \\
4 \\
4 \\
2\end{array}$ & $\begin{array}{l}6 \\
6 \\
6 \\
6 \\
6 \\
4 \\
2 \\
2\end{array}$ & $\begin{array}{r}8 \\
8 \\
10 \\
6 \\
8 \\
8 \\
4 \\
4\end{array}$ & $\begin{array}{l}3 \\
2 \\
4 \\
3 \\
1 \\
2 \\
2 \\
1\end{array}$ & $\begin{array}{l}18 \\
14 \\
24 \\
14 \\
10 \\
34 \\
6 \\
8\end{array}$ & $\begin{array}{r}16 \\
10 \\
46 \\
12 \\
6 \\
54 \\
2 \\
4\end{array}$ & $\begin{array}{l}2241 \\
2212 \\
2558 \\
2156 \\
2011 \\
1898 \\
2219 \\
2377\end{array}$ & $\begin{array}{r}10 \\
22 \\
24 \\
8 \\
4 \\
70 \\
4 \\
3\end{array}$ & $\begin{array}{l}1296 \\
1733 \\
7414 \\
1795 \\
1588 \\
6905 \\
1783 \\
6428\end{array}$ & $\begin{array}{l}37 \\
39 \\
64 \\
48 \\
48 \\
49 \\
46 \\
49\end{array}$ & $\begin{array}{l}112 \\
127 \\
139 \\
117 \\
118 \\
101 \\
110 \\
134\end{array}$ & $\begin{array}{l}27 \\
20 \\
45 \\
17 \\
17 \\
41 \\
17 \\
39\end{array}$ \\
\hline
\end{tabular}

Notes: $\mathrm{g}=$ gabbro, $\mathrm{BS}=$ basalt, $\mathrm{tr}=$ troctolite, $\mathrm{hz}=$ harzburgite, $\mathrm{d}=$ dunite. Zero $=$ not detected, blank $=$ not analyzed.

\section{Platinum-Group-Element Concentrations}

The greatest concentrations of PGE were found in both the troctolites and dunites. The two samples containing the most anomalous PGE concentrations are troctolites (data set 1, Table 1) with 106 and $99 \mathrm{ppb}$ total PGE in Samples 147-895E-6R-1, (Piece 17, 90-92 cm) and 147-895D-7R-1, (Piece 8, 45-49 cm), respectively. The troctolite section from Section 147-895D-7R-1 (Piece 7 through Piece 16) has anomalous sulfide and chrome-spinel concentrations. The troctolite section contains one of the most anomalous PGE values in Sample 147-895D-7R-1 (Piece 8, 45-49 cm) and a lower but still anomalous value of $31 \mathrm{ppb}$ total PGE in Sample 147-895D-7R-1, (Piece 12, 74-79 cm). The more anomalous sample is from the uppermost part of the troctolite section and the sample with the lower value is from the center. Thus, it may be inferred that the PGE content increases upward in this section of troctolite in conjunction with the increases in sulfide and chrome-spinel. In addition to the troctolites, one sulfide-bearing, chromite-bearing dunite, Sample 147-895E-4R1 (Piece 19, 131-134 cm), contained $96 \mathrm{ppb}$ total PGE.

The relative proportions of the PGE can be examined using chondrite-normalized plots (Fig. 4). PGE analyses with relatively more Os, Ir, and Ru than Pt and Pd have negatively sloping chondrite-normalized profiles that usually indicate a chromite association whereas $\mathrm{Pt}$ and Pd enrichment results in positively sloping chondrite-normalized profiles that commonly indicate a sulfide association. In Hess Deep, samples with the greatest PGE have slightly positive slope profiles. All the sulfur-bearing troctolites analyzed have positively sloping profiles and the most enriched sulfur-bearing dunite also has a positively sloping profile. Although troctolites from data set 2 (Table 2) and Sample 147-895C-4R-1 (Piece 6C, 129-133 cm) from data set 1 (Table 1) also have positive chondrite-normalized profiles, they show a slight positive anomaly at Ru. This may reflect a slight enrichment of Ru in the unusually large (typically approximately $1 \mathrm{~cm}$ in diameter) chrome-spinels common in these troctolites. Some of the dunites have slight $\mathrm{Ru}$ anomalies but they generally have variable chondrite-normalized profiles; with low PGE concentrations, small differences in the PGE values result in both slightly positive and slightly negative slope profiles. The chondrite-normalized profiles for the harzburgite, from both data sets, show a sigmoidal pattern with peaks at $\mathrm{Ru}$ and $\mathrm{Pd}$.

The samples analyzed for PGE in data set 2 (Table 2) were also analyzed for sulfur (Puchelt et al.,this volume). This enabled calculation of the PGE values in $100 \%$ of sulfide, which were plotted for harzburgite, dunite, and gabbro on chondrite-normalized profiles (Fig. 5). All three lithologies have slightly sigmoidal patterns with enrichment in $\mathrm{Ru}$ and $\mathrm{Pd}$. These patterns are similar to those for whole-rock chondrite-normalized profiles for harzburgites, dunites, and gabbros that have low PGE concentrations. However, the abundance of PGE in the sulfide varies, and it is clear that the values for PGE in $100 \%$ of sulfide are slightly higher for harzburgite than for dunite or gabbro.

The common association of anomalous $\mathrm{Pt}$ and $\mathrm{Pd}$ with enriched base metals is confirmed in these samples. For example, the dunite with the most anomalous $\mathrm{Pt}$ and $\mathrm{Pd}$ values also has distinctly greater values of $\mathrm{Ni}, \mathrm{Cu}, \mathrm{Zn}, \mathrm{Co}$, and $\mathrm{V}$ compared to the other dunites and it also has the greatest value of $\mathrm{Cr}$ (Fig. 6). Coincidences of anomalous PGE and base metals in the troctolites are, in general, less apparent. This can be explained by the more complicated silicate mineralogy (dunites are virtually monomineralic whereas the troctolites contain varying quantities of olivine and the $\mathrm{Ni}$ content will be dependent on the quantity of olivine as well as the sulfide). Although the trend of increasing base metal and $\mathrm{Cr}$ element abundances upsection is not perfect in the troctolite Section 147-895D-7R-1 (Pieces 7 through 16), the greater values in the upper samples compared to the lower samples tend to confirm the observation made during core logging of an increasing abundance of sulfide and chrome-spinel toward the top of the troctolite. This coincides with increasing PGE values upward (Table 6). It should be noted that the geochemical data only partially support the shipboard observations and are of limited use because of (1) the incomplete sampling due to sampling restrictions and (2) the possibility of unrepresentative concentrations due to the coarse crystal size of the troctolite and small sample size homogenized for analysis. A more comprehensive analysis of the troctolite core section that would confirm or disprove these trends was not possible for this study owing to initial sampling restrictions on board. 
Table 2. PGE analyses for samples from Sites 894 and 895 , data set 2.

\begin{tabular}{|c|c|c|c|c|c|c|c|c|c|c|c|c|c|c|c|c|c|c|c|c|c|c|c|}
\hline $\begin{array}{l}\text { Core, section, } \\
\text { interval }(\mathrm{cm})\end{array}$ & $\begin{array}{l}\text { Depth } \\
\text { (mbsf) }\end{array}$ & Lithology & Os & Ir & $\mathrm{Ru}$ & $\mathrm{Rh}$ & $\mathrm{Pt}$ & Pd & $\mathrm{Au}$ & $\begin{array}{c}\mathrm{Ni} \\
\text { (INAA) }\end{array}$ & $\begin{array}{c}\mathrm{Ni} \\
(\mathrm{XRF})\end{array}$ & $\mathrm{Cu}$ & $\mathrm{Cr}$ & $\begin{array}{c}\mathrm{Zn} \\
\text { (INAA) }\end{array}$ & $\begin{array}{c}\mathrm{Zn} \\
\text { (XRF) }\end{array}$ & Co & $\mathrm{Sc}$ & $\mathrm{Sr}$ & $\mathrm{Pb}$ & $\mathrm{Mn}$ & $\underset{(\%)}{\mathrm{Na}_{2} \mathrm{O}}$ & $\begin{array}{c}\mathrm{Fe}_{2} \mathrm{O}_{3} \\
\text { (INAA) } \\
(\%)\end{array}$ & $\begin{array}{l}\mathrm{Fe}_{2} \mathrm{O}_{3} \\
\text { (XRF) } \\
\text { (\%) }\end{array}$ \\
\hline $\begin{array}{l}\text { 147-894F- } \\
\text { 3R-1 (Piece 17, 98-103) }\end{array}$ & 17.79 & g & 0.0 & 0.0 & 2.4 & 1.5 & 7.0 & 0.0 & 0.0 & 112 & 107 & 62 & 664 & 38 & 53 & 41 & 43 & 96 & 0 & 0.2 & 2.28 & 7.7 & 8.0 \\
\hline 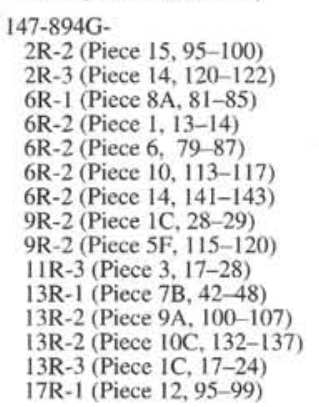 & $\begin{array}{r}31.05 \\
32.75 \\
55.61 \\
56.35 \\
57.02 \\
57.36 \\
57.64 \\
75.93 \\
76.80 \\
87.14 \\
103.90 \\
105.80 \\
106.10 \\
106.40 \\
126.80\end{array}$ & $\begin{array}{l}\text { BS } \\
\text { ol g } \\
\text { gn } \\
\text { gn } \\
\mathrm{gn} \\
\mathrm{gn} \\
\mathrm{gn} \\
\mathrm{gn} \\
\mathrm{gn} \\
\mathrm{gn} \\
\mathrm{gn} \\
\mathrm{gn} \\
\mathrm{gn} \\
\mathrm{gn} \\
\mathrm{gn}\end{array}$ & $\begin{array}{l}0.0 \\
0.0 \\
0.0 \\
0.0 \\
0.0 \\
0.9 \\
0.0 \\
0.0 \\
0.0 \\
0.0 \\
0.0 \\
0.0 \\
0.0 \\
0.0 \\
0.0\end{array}$ & $\begin{array}{l}0.0 \\
0.0 \\
0.0 \\
0.0 \\
0.5 \\
0.0 \\
0.0 \\
0.0 \\
0.0 \\
0.0 \\
0.0 \\
0.0 \\
0.4 \\
0.2 \\
0.0\end{array}$ & $\begin{array}{l}3.8 \\
0.0 \\
0.0 \\
2.4 \\
3.1 \\
1.7 \\
4.7 \\
0.0 \\
4.3 \\
1.8 \\
2.3 \\
1.4 \\
0.0 \\
4.0 \\
0.0\end{array}$ & $\begin{array}{l}0.0 \\
0.0 \\
0.0 \\
0.0 \\
0.0 \\
0.0 \\
0.0 \\
0.0 \\
0.0 \\
0.0 \\
0.0 \\
0.0 \\
2.1 \\
0.0 \\
0.0\end{array}$ & $\begin{array}{l}4.7 \\
2.1 \\
0.0 \\
0.0 \\
2.4 \\
4.2 \\
0.0 \\
0.0 \\
0.0 \\
1.2 \\
0.0 \\
0.0 \\
3.2 \\
7.4 \\
0.0\end{array}$ & $\begin{array}{l}1.8 \\
0.0 \\
0.0 \\
0.0 \\
3.5 \\
0.0 \\
1.6 \\
0.0 \\
1.9 \\
0.0 \\
1.6 \\
0.0 \\
1.5 \\
4.2 \\
0.0\end{array}$ & $\begin{array}{l}1.7 \\
0.0 \\
1.2 \\
1.9 \\
1.6 \\
0.0 \\
2.1 \\
0.0 \\
3.1 \\
0.0 \\
1.8 \\
0.0 \\
1.0 \\
0.0 \\
0.0\end{array}$ & $\begin{array}{r}204 \\
75 \\
40 \\
73 \\
100 \\
73 \\
87 \\
83 \\
65 \\
78 \\
66 \\
101 \\
96 \\
105 \\
106\end{array}$ & $\begin{array}{r}193 \\
177 \\
20 \\
84 \\
94 \\
101 \\
102 \\
65 \\
67 \\
88 \\
67 \\
63 \\
84 \\
74 \\
93\end{array}$ & $\begin{array}{l}79 \\
93 \\
41 \\
90 \\
84 \\
77 \\
55 \\
19 \\
20 \\
69 \\
33 \\
75 \\
41 \\
75 \\
92\end{array}$ & $\begin{array}{l}323 \\
372 \\
123 \\
118 \\
335 \\
308 \\
260 \\
122 \\
149 \\
170 \\
211 \\
246 \\
218 \\
312 \\
399\end{array}$ & $\begin{array}{l}46 \\
56 \\
58 \\
81 \\
44 \\
75 \\
35 \\
43 \\
41 \\
88 \\
26 \\
54 \\
51 \\
37 \\
51\end{array}$ & $\begin{array}{l}55 \\
73 \\
61 \\
66 \\
49 \\
66 \\
50 \\
53 \\
44 \\
73 \\
46 \\
52 \\
67 \\
49 \\
50\end{array}$ & $\begin{array}{l}42 \\
36 \\
34 \\
46 \\
41 \\
42 \\
47 \\
36 \\
30 \\
48 \\
32 \\
38 \\
48 \\
38 \\
39\end{array}$ & $\begin{array}{l}30 \\
46 \\
47 \\
46 \\
53 \\
56 \\
54 \\
41 \\
40 \\
55 \\
50 \\
46 \\
49 \\
45 \\
55\end{array}$ & $\begin{array}{r}85 \\
88 \\
97 \\
83 \\
86 \\
85 \\
73 \\
106 \\
117 \\
83 \\
99 \\
95 \\
76 \\
92 \\
86\end{array}$ & $\begin{array}{r}0 \\
13 \\
0 \\
0 \\
0 \\
0 \\
0 \\
0 \\
0 \\
0 \\
0 \\
0 \\
0 \\
18 \\
0\end{array}$ & $\begin{array}{l}0.2 \\
0.2 \\
0.2 \\
0.2 \\
0.2 \\
0.2 \\
0.2 \\
0.2 \\
0.1 \\
0.2 \\
0.2 \\
0.2 \\
0.2 \\
0.2 \\
0.2\end{array}$ & $\begin{array}{l}2.18 \\
2.95 \\
3.17 \\
2.68 \\
2.33 \\
2.52 \\
2.38 \\
3.08 \\
3.38 \\
2.51 \\
2.75 \\
2.49 \\
2.27 \\
2.46 \\
2.04\end{array}$ & $\begin{array}{r}8.4 \\
7.9 \\
8.7 \\
11.3 \\
8.7 \\
9.7 \\
9.9 \\
8.7 \\
7.6 \\
11.6 \\
7.9 \\
8.8 \\
10.7 \\
8.2 \\
8.4\end{array}$ & $\begin{array}{r}8.7 \\
11.8 \\
8.9 \\
12.4 \\
8.8 \\
9.5 \\
10.3 \\
9.0 \\
7.6 \\
12.3 \\
7.9 \\
8.7 \\
11.7 \\
7.7 \\
8.1\end{array}$ \\
\hline $\begin{array}{l}\text { 147-895A- } \\
\text { 2R-1 (Piece 4, 19-26) } \\
\text { 2R-1 (Piece 4, 19-26) } \\
\text { 2R-1 (Piece 9B, 19-26) }\end{array}$ & $\begin{array}{l}9.36 \\
9.36 \\
9.36\end{array}$ & $\begin{array}{l}\text { BS } \\
\text { BS } \\
\mathrm{hz} / \mathrm{s}\end{array}$ & $\begin{array}{l}0.0 \\
0.0 \\
0.0\end{array}$ & $\begin{array}{l}0.0 \\
0.0 \\
0.0\end{array}$ & $\begin{array}{l}0.0 \\
0.0 \\
0.0\end{array}$ & $\begin{array}{l}0.0 \\
0.0 \\
2.3\end{array}$ & $\begin{array}{l}0.0 \\
5.6 \\
2.2\end{array}$ & $\begin{array}{l}0.0 \\
0.0 \\
0.0\end{array}$ & $\begin{array}{l}0.0 \\
1.8 \\
2.4\end{array}$ & $\begin{array}{r}36 \\
36 \\
2007\end{array}$ & $\begin{array}{r}20 \\
20 \\
1861\end{array}$ & $\begin{array}{l}25 \\
25 \\
60\end{array}$ & $\begin{array}{r}28 \\
28 \\
2822\end{array}$ & $\begin{array}{r}135 \\
135 \\
42\end{array}$ & $\begin{array}{r}123 \\
123 \\
48\end{array}$ & $\begin{array}{r}37 \\
37 \\
102\end{array}$ & $\begin{array}{l}44 \\
44 \\
9.6\end{array}$ & $\begin{array}{r}112 \\
112 \\
0\end{array}$ & $\begin{array}{r}0 \\
0 \\
10\end{array}$ & $\begin{array}{l}0.4 \\
0.4 \\
0.2\end{array}$ & $\begin{array}{l}4.15 \\
4.15 \\
0.48\end{array}$ & $\begin{array}{r}15.2 \\
15.2 \\
8.2\end{array}$ & $\begin{array}{r}17.9 \\
17.9 \\
8.6\end{array}$ \\
\hline $\begin{array}{l}\text { 147-895D- } \\
\text { 3R-1 (Piece 13, 108-111) } \\
\text { 4R-4 (Piece 12, 87-91) } \\
\text { 6R-1 (Piece 17, 128-134) } \\
7 \mathrm{R}-1 \text { (Piece 8, 45-49) }\end{array}$ & $\begin{array}{l}27.08 \\
39.32 \\
56.30 \\
65.05\end{array}$ & $\begin{array}{l}\mathrm{hz} \\
\mathrm{hz} / \mathrm{s} \\
\mathrm{hz} \\
\mathrm{tr}\end{array}$ & $\begin{array}{l}0.0 \\
0.7 \\
0.0 \\
0.0\end{array}$ & $\begin{array}{l}1.3 \\
1.7 \\
2.6 \\
1.2\end{array}$ & $\begin{array}{l}2.9 \\
4.3 \\
10.5 \\
7.0\end{array}$ & $\begin{array}{l}0.0 \\
0.0 \\
1.5 \\
0.0\end{array}$ & $\begin{array}{r}1.7 \\
2.8 \\
9.3 \\
14.1\end{array}$ & $\begin{array}{r}1.9 \\
6.2 \\
8.6 \\
20.9\end{array}$ & $\begin{array}{l}0.0 \\
2.6 \\
2.6 \\
6.7\end{array}$ & $\begin{array}{l}2183 \\
2016 \\
2055 \\
2608\end{array}$ & $\begin{array}{l}2088 \\
2041 \\
1876 \\
2217\end{array}$ & $\begin{array}{r}26 \\
25 \\
47 \\
324\end{array}$ & $\begin{array}{l}2548 \\
2625 \\
3360 \\
8573\end{array}$ & $\begin{array}{l}48 \\
36 \\
43 \\
62\end{array}$ & $\begin{array}{l}55 \\
48 \\
39 \\
45\end{array}$ & $\begin{array}{l}110 \\
100 \\
107 \\
116\end{array}$ & $\begin{array}{c}9.6 \\
9.3 \\
12 \\
4.8\end{array}$ & $\begin{array}{r}0 \\
79 \\
0 \\
0\end{array}$ & $\begin{array}{r}0 \\
0 \\
27 \\
0\end{array}$ & $\begin{array}{l}0.2 \\
0.2 \\
0.2 \\
0.3\end{array}$ & $\begin{array}{l}0.05 \\
0.06 \\
0.07 \\
0.07\end{array}$ & $\begin{array}{l}8.2 \\
8.0 \\
8.0 \\
9.2\end{array}$ & $\begin{array}{r}9.6 \\
9.3 \\
8.7 \\
10.1\end{array}$ \\
\hline $\begin{array}{l}\text { 147-895E- } \\
\text { 1R-1 (Piece 7A, 64-68) } \\
\text { IR-3 (Piece 2A, 20-24) } \\
\text { IR-3 (Piece 3A, 51-55) } \\
\text { IR-3 (Piece 4B, 62-64) } \\
\text { 4R-1 (Piece 6, 29-31) } \\
\text { 4R-1 (Piece 7, 34-36) } \\
\text { 4R-1 (Piece 17, 117-126) } \\
\text { 6R-1 (Piece 21, 110-114) } \\
\text { 7R-2 (Piece 5, 14-49) } \\
\text { 8R-4 (Piece 6, 28-35) }\end{array}$ & $\begin{array}{r}0.64 \\
3.06 \\
3.37 \\
3.48 \\
39.79 \\
39.84 \\
40.67 \\
59.70 \\
70.14 \\
82.58\end{array}$ & $\begin{array}{l}\text { ol g } \\
\mathrm{hz} / \mathrm{s} \\
\mathrm{d} / \mathrm{s} \\
\mathrm{hz} / \mathrm{s} \\
\mathrm{tr} \\
\mathrm{tr} \\
\mathrm{h} / \mathrm{s} \\
\mathrm{d} / \mathrm{s} \\
\mathrm{d} \\
\mathrm{d}\end{array}$ & $\begin{array}{l}0.0 \\
0.0 \\
0.8 \\
0.0 \\
0.0 \\
0.0 \\
2.4 \\
0.0 \\
0.9 \\
0.0\end{array}$ & $\begin{array}{l}0.0 \\
2.4 \\
2.8 \\
1.5 \\
0.4 \\
0.3 \\
2.5 \\
1.4 \\
2.6 \\
2.2\end{array}$ & $\begin{array}{l}1.8 \\
5.0 \\
6.4 \\
5.7 \\
4.0 \\
0.0 \\
6.5 \\
2.9 \\
5.0 \\
4.8\end{array}$ & $\begin{array}{l}0.0 \\
1.2 \\
1.3 \\
0.0 \\
0.0 \\
1.2 \\
0.0 \\
0.0 \\
1.4 \\
0.0\end{array}$ & $\begin{array}{l}2.0 \\
5.6 \\
6.9 \\
2.7 \\
4.0 \\
2.2 \\
5.9 \\
5.3 \\
8.0 \\
6.7\end{array}$ & $\begin{array}{r}12.5 \\
8.0 \\
8.5 \\
0.0 \\
6.2 \\
3.4 \\
6.8 \\
1.9 \\
3.3 \\
5.4\end{array}$ & $\begin{array}{l}1.6 \\
3.1 \\
2.9 \\
1.6 \\
1.6 \\
2.9 \\
2.4 \\
3.7 \\
2.6 \\
3.1\end{array}$ & $\begin{array}{r}776 \\
2224 \\
2223 \\
2276 \\
1480 \\
1718 \\
2127 \\
2125 \\
2139 \\
2000\end{array}$ & $\begin{array}{r}802 \\
2024 \\
2354 \\
2229 \\
1442\end{array}$ & $\begin{array}{r}18 \\
48 \\
25 \\
58 \\
117\end{array}$ & $\begin{array}{l}1044 \\
2190 \\
3302 \\
1840 \\
2227 \\
6657 \\
3176 \\
2260 \\
2925 \\
3021\end{array}$ & $\begin{array}{l}31 \\
46 \\
46 \\
42 \\
33 \\
48 \\
52 \\
45 \\
49 \\
50\end{array}$ & $\begin{array}{l}39 \\
42 \\
46 \\
48 \\
25\end{array}$ & $\begin{array}{r}60 \\
110 \\
120 \\
115 \\
117 \\
111 \\
115 \\
126 \\
121 \\
126\end{array}$ & $\begin{array}{l}2.7 \\
8.9 \\
8.0 \\
8.2 \\
3.9 \\
4.1 \\
4.9 \\
4.9 \\
5.0 \\
5.2\end{array}$ & $\begin{array}{r}134 \\
0 \\
0 \\
8 \\
12\end{array}$ & $\begin{array}{l}0 \\
0 \\
0 \\
0 \\
0\end{array}$ & $\begin{array}{l}0.1 \\
0.2 \\
0.2 \\
0.1 \\
0.1\end{array}$ & $\begin{array}{c}0.77 \\
0.03 \\
0.04 \\
0.04 \\
0.10 \\
0.07 \\
0.05 \\
10.3 \\
0.04 \\
0.04\end{array}$ & $\begin{array}{l}5.4 \\
8.6 \\
9.7 \\
8.9 \\
9.0 \\
8.4 \\
9.5\end{array}$ & $\begin{array}{r}5.5 \\
9.2 \\
11.2 \\
9.1 \\
10.0 \\
11.5 \\
12.3 \\
12.4\end{array}$ \\
\hline $\begin{array}{l}\text { 147-895F- } \\
\text { 2R-1 (Piece 8A, 65-69) } \\
\text { 2R-2 (Piece 8, 66-71) }\end{array}$ & $\begin{array}{l}18.85 \\
20.36\end{array}$ & $\begin{array}{l}\mathrm{hz} / \mathrm{s} \\
\mathrm{hz} / \mathrm{s}\end{array}$ & $\begin{array}{l}0.0 \\
0.0\end{array}$ & $\begin{array}{l}1.6 \\
2.3\end{array}$ & $\begin{array}{l}6.0 \\
4.9\end{array}$ & $\begin{array}{l}0.0 \\
0.0\end{array}$ & $\begin{array}{l}5.8 \\
5.5\end{array}$ & $\begin{array}{l}4.4 \\
7.4\end{array}$ & $\begin{array}{l}0.0 \\
0.0\end{array}$ & $\begin{array}{l}2074 \\
1968\end{array}$ & $\begin{array}{l}2038 \\
1829\end{array}$ & $\begin{array}{l}24 \\
22\end{array}$ & $\begin{array}{l}1418 \\
2748\end{array}$ & $\begin{array}{l}31 \\
46\end{array}$ & $\begin{array}{l}36 \\
47\end{array}$ & $\begin{array}{l}101 \\
109\end{array}$ & $\begin{array}{r}8.4 \\
12.0\end{array}$ & $\begin{array}{l}0 \\
7\end{array}$ & $\begin{array}{r}11 \\
0\end{array}$ & $\begin{array}{l}0.1 \\
0.2\end{array}$ & $\begin{array}{l}0.1 \\
0.04\end{array}$ & $\begin{array}{l}7.9 \\
8.3\end{array}$ & $\begin{array}{l}8.8 \\
9.5\end{array}$ \\
\hline
\end{tabular}

Notes: PGE Os, Ir, Ru, Rh, Pt, and Pd in ppb; pathfinder elements Ni, Cu, Cr, $\mathrm{Zn}, \mathrm{Co}, \mathrm{V}, \mathrm{Sc}, \mathrm{Sr}$, and $\mathrm{Pb}$ in ppm; $\mathrm{Mn}, \mathrm{Fe}_{2} \mathrm{O}_{3}$, and $\mathrm{Na}_{2} \mathrm{O}$ in weight percent. Lithology: $\mathrm{g}=$ gabbro, $\mathrm{BS}=$ basalt, ol $\mathrm{g}=$ olivine gabbro, gn = gabbronorite, hz = harzburgite, $\mathrm{s}=$ serpentinite, $\mathrm{tr}=$ troctolite, $\mathrm{d}=$ dunite. Zero $=$ not detected, blank $=$ not analyzed. 
Table 3. Comparison of PGE values (ppb) from data sets 1 and 2.

\begin{tabular}{|c|c|c|c|c|c|c|c|c|}
\hline $\begin{array}{l}\text { Core, section, } \\
\text { interval }(\mathrm{cm})\end{array}$ & $\begin{array}{c}\text { Data } \\
\text { set }\end{array}$ & Lithology & Os & Ir & Ru & $\mathrm{Rh}$ & $\mathrm{Pt}$ & Pd \\
\hline \multicolumn{9}{|l|}{$\begin{array}{l}\text { 147-894G- } \\
\text { 13R-1 (Piece 7B, 42-48) }\end{array}$} \\
\hline 13R-1 (Piece 7B, 42-48) & $\begin{array}{l}1 \\
2\end{array}$ & g & $\frac{2}{0}$ & $\begin{array}{l}2 \\
0\end{array}$ & $\begin{array}{l}0 \\
2.3\end{array}$ & $\begin{array}{l}1 \\
0\end{array}$ & $\begin{array}{l}4 \\
0\end{array}$ & $\begin{array}{l}0 \\
1.6\end{array}$ \\
\hline 13R-2 (Piece 9A, 100-107) & $\begin{array}{l}1 \\
2\end{array}$ & $\mathrm{~g}$ & $\begin{array}{l}2 \\
0\end{array}$ & $\begin{array}{l}2 \\
0\end{array}$ & $\begin{array}{l}2 \\
1.4\end{array}$ & $\begin{array}{l}1 \\
0\end{array}$ & $\begin{array}{l}4 \\
0\end{array}$ & $\begin{array}{l}0 \\
0\end{array}$ \\
\hline \multirow{2}{*}{$\begin{array}{l}\text { 147-895D- } \\
\text { 7R-1 (Piece 8, 45-49) }\end{array}$} & & & & & & & & \\
\hline & 1 & tr & 4 & 4 & 8 & 3 & 36 & 44 \\
\hline
\end{tabular}

Notes: $\mathrm{g}=$ gabbro, $\mathrm{tr}=$ troctolite. Zero $=$ not detected.

Table 4. PGE analyses (ppb) from a core of sedimentary material derived from serpentinite.

\begin{tabular}{|c|c|c|c|c|c|c|c|c|c|c|c|c|}
\hline Analysis & Os & Ir & $\mathrm{Ru}$ & $\mathrm{Rh}$ & $\mathrm{Pt}$ & $\mathrm{Pd}$ & $\mathrm{Au}$ & $\mathrm{Ni}$ & $\mathrm{Cr}$ & $\mathrm{Zn}$ & Co & $\mathrm{Fe}_{2} \mathrm{O}_{3}$ \\
\hline 1 & 0 & 1.3 & 2.9 & 0 & 1.7 & 1.9 & 0 & 2183 & 2548 & 48 & 110 & 8.2 \\
\hline 18 & 0 & 0.4 & 0 & 2.1 & 3.2 & 1.5 & 1.0 & 96 & 218 & 51 & 48 & 10.7 \\
\hline 19 & 0 & 0 & 2.4 & 0 & 0 & 0 & 1.9 & 73 & 118 & 81 & 46 & 11.3 \\
\hline 20 & 0.7 & 1.7 & 4.3 & 0 & 2.8 & 6.2 & 2.6 & 2016 & 2625 & 36 & 100 & 8.0 \\
\hline 23 & 0 & 0 & 0 & 0 & 0 & 0 & 0 & 106 & 399 & 51 & 39 & 8.4 \\
\hline 24 & 0 & 0 & 0 & 0 & 0 & 0 & 1.2 & 40 & 123 & 58 & 34 & 8.7 \\
\hline 25 & 0.9 & 0 & 1.7 & 0 & 4.2 & 0 & 0 & 73 & 308 & 75 & 42 & 9.7 \\
\hline 26 & 0 & 0 & 0 & 0 & 2.1 & 0 & 0 & 75 & 372 & 56 & 36 & 7.9 \\
\hline 32 & 0 & 0 & 0 & 0 & 5.6 & 0 & 1.8 & 36 & 28 & 135 & 37 & 15.2 \\
\hline 34 & 0 & 0 & 0 & 2.3 & 2.2 & 0 & 2.4 & 2007 & 2822 & 42 & 102 & 8.2 \\
\hline 342 & 2.4 & 2.5 & 6.5 & 0 & 5.9 & 6.8 & 2.4 & 2127 & 3176 & 52 & 115 & 9.5 \\
\hline 39 & 0 & 0.3 & 0 & 1.2 & 2.2 & 3.4 & 2.9 & 1718 & 6657 & 48 & 111 & 8.4 \\
\hline 4 & 0 & 0 & 0 & 0 & 0 & 0 & 0 & 83 & 122 & 43 & 36 & 8.7 \\
\hline 42 & 0 & 2.3 & 4.9 & 0 & 5.5 & 7.4 & 0 & 1968 & 2748 & 46 & 109 & 8.3 \\
\hline 45 & 0 & 1.4 & 2.9 & 0 & 5.3 & 1.9 & 3.7 & 2125 & 2260 & 45 & 126 & 10.3 \\
\hline 47 & 0.9 & 2.6 & 5 & 1.4 & 8.0 & 3.3 & 2.6 & 2139 & 2925 & 49 & 121 & 10.3 \\
\hline 49 & 0.8 & 2.8 & 6.4 & 1.3 & 6.9 & 8.5 & 2.9 & 2223 & 3302 & 46 & 120 & 9.7 \\
\hline 50 & 0 & 2.2 & 4.8 & 0 & 6.7 & 5.4 & 3.1 & 2000 & 3021 & 50 & 126 & 10.3 \\
\hline 54 & 0 & 2.4 & 5.0 & 1.2 & 5.6 & 8.0 & 3.1 & 2224 & 2190 & 46 & 110 & 8.6 \\
\hline 6 & 0 & 0 & 4.3 & 0 & 0 & 1.9 & 3.1 & 65 & 149 & 41 & 30 & 7.6 \\
\hline
\end{tabular}

Table 5. PGM analyses in weight percent.

\begin{tabular}{|c|c|c|c|c|}
\hline $\begin{array}{l}\text { Sample } 147-895 \mathrm{E}-6 \mathrm{R}-1 \\
\text { (Piece } 17,90-92 \mathrm{~cm} \text { ) }\end{array}$ & $\begin{array}{l}\mathrm{Pd}-\mathrm{Sn}-\mathrm{Cu} \\
\text { (1) }\end{array}$ & $\begin{array}{c}\mathrm{Pt}-\mathrm{Fe}-\mathrm{Ni}-\mathrm{Cu} \\
\text { (2) }\end{array}$ & $\begin{array}{c}\mathrm{Cu}-\mathrm{Pt}-\mathrm{Au} \\
\text { (3) }\end{array}$ & $\begin{array}{l}\text { Pt-Ni-Cu } \\
\text { (4) }\end{array}$ \\
\hline $\begin{array}{l}\mathrm{Fe} \\
\mathrm{Ni} \\
\mathrm{Cu} \\
\mathrm{Sn} \\
\mathrm{Pd} \\
\mathrm{Pt} \\
\mathrm{Au}\end{array}$ & $\begin{array}{r}0.82 \\
2.79 \\
16.18 \\
30.86 \\
50.22\end{array}$ & $\begin{array}{r}19.18 \\
14.84 \\
14.06 \\
0.93 \\
\\
51.45\end{array}$ & $\begin{array}{r}2.33 \\
3.62 \\
65.23 \\
\\
0.62 \\
21.12 \\
6.97\end{array}$ & $\begin{array}{l}13.01 \\
22.51 \\
18.07\end{array}$ \\
\hline Total & $\begin{array}{c}100.87 \\
\mathrm{Ni}-\mathrm{Sn} \\
(5)\end{array}$ & $\begin{array}{c}100.46 \\
\begin{array}{c}\text { Pt-Ni-Fe } \\
(6)\end{array}\end{array}$ & $\begin{array}{c}99.89 \\
\begin{array}{c}\text { Ni-Pt-Fe } \\
(7)\end{array}\end{array}$ & $\begin{array}{c}97.17 \\
\mathrm{Pt}-\mathrm{Fe} \\
(8)\end{array}$ \\
\hline $\begin{array}{l}\mathrm{Fe} \\
\mathrm{Ni} \\
\mathrm{Cu} \\
\mathrm{Sn} \\
\mathrm{Pd} \\
\mathrm{Pt}\end{array}$ & $\begin{array}{r}1.64 \\
53.84 \\
4.00 \\
36.63 \\
0.57 \\
2.93\end{array}$ & $\begin{array}{r}18.17 \\
34.24 \\
1.22 \\
\\
45.26\end{array}$ & $\begin{array}{r}18.38 \\
45.32 \\
4.78 \\
3.04 \\
5.41 \\
22.80\end{array}$ & $\begin{array}{r}15.00 \\
6.60 \\
5.17 \\
0.53 \\
\\
72.57\end{array}$ \\
\hline Total & 99.61 & 98.89 & 99.73 & 99.87 \\
\hline $\begin{array}{l}\text { Sample 147-895D-7R-1 } \\
\text { (Piece } 8,45-49 \mathrm{~cm} \text { ) }\end{array}$ & $\begin{array}{c}\text { Bi-Te-Pd-Pt } \\
(9)\end{array}$ & & & \\
\hline $\begin{array}{l}\mathrm{S} \\
\mathrm{Fe} \\
\mathrm{Ni} \\
\mathrm{Cu} \\
\mathrm{Bi} \\
\mathrm{Pd} \\
\mathrm{Te} \\
\mathrm{Pt}\end{array}$ & $\begin{array}{r}12.15 \\
13.94 \\
10.91 \\
2.71 \\
27.06 \\
8.98 \\
20.58 \\
3.82\end{array}$ & & & \\
\hline Total & 100.15 & & & \\
\hline
\end{tabular}

Notes: Numbers (1)-(9) correspond to labels in Figure 10. Blank $=$ not detected. 


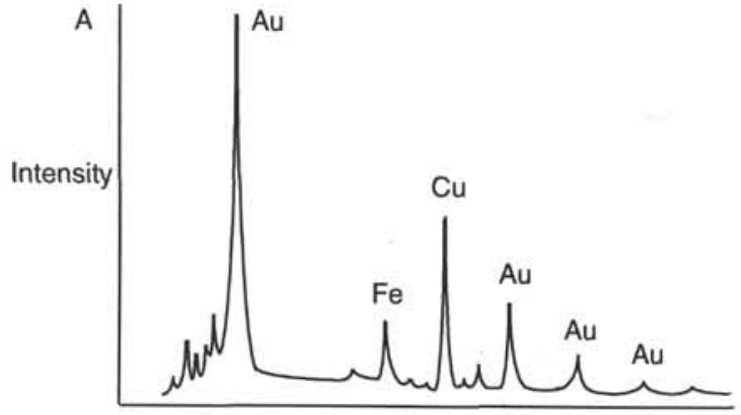

Energy

B

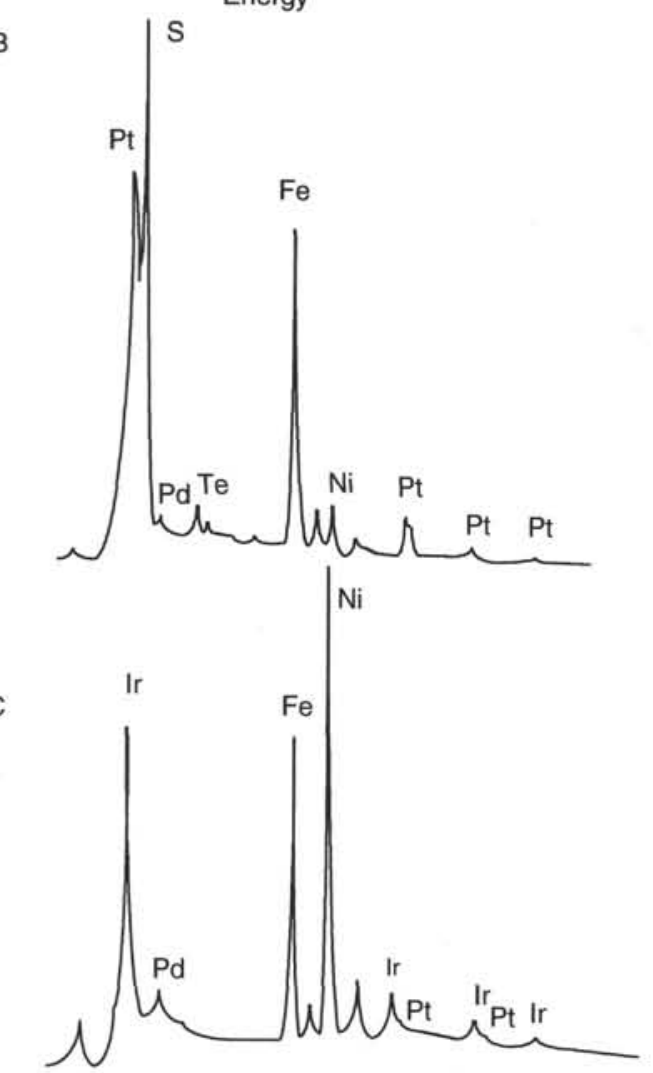

Figure 3. Energy-dispersive X-ray spectra for precious metals too small for quantitative analysis: (A) Cu-Au alloy from Sample 147-895D-7R-1 (Piece 11, 71-74 cm), (B) Pt-S from Sample 147-895D-7R-1 (Piece 8, 45-49 cm), and (C) Ir-Pt alloy from Sample 147-895E-4R-1 (Piece 19, 131-134 cm).

The association of PGE with base metals also has been observed in the gabbros, where although the PGE concentrations are lower, $\mathrm{Ni}$, $\mathrm{Cu}$, and $\mathrm{Cr}$ increase with increasing $\mathrm{Pt}+\mathrm{Pd}$ (Fig. 7).

\section{Rare Earth Elements and Platinum-Group Elements}

REE were analyzed for all the samples in data set 2 (Table 2). The REE values for the ultramafic lithologies for Site 895 are characteristically small, commonly below detection. In the gabbro from Site 894 , both REE and PGE values are sufficiently great for correlations to be sought (Table 7). This reveals that $(\mathrm{Pt}+\mathrm{Pd})$-enriched and -depleted samples both show depletion in the light REE (LREE) relative to the heavy REE (HREE) but that the ( $\mathrm{Pt}+\mathrm{Pd}$ )-enriched samples are more depleted in LREE (Fig. 8). This is emphasized by a graph of $\mathrm{Pt}$ $+\mathrm{Pd}$ versus $\mathrm{La}+\mathrm{Ce}$, which shows a negative slope (Fig. 9). In addition, $(\mathrm{Pt}+\mathrm{Pd})$-enriched gabbros are less enriched in both LREE and
HREE than $(\mathrm{Pt}+\mathrm{Pd})$-poor gabbros, and $(\mathrm{Pt}+\mathrm{Pd})$-enriched gabbros have no Eu anomaly whereas the most $(\mathrm{Pt}+\mathrm{Pd})$-poor gabbros have a slight negative anomaly.

\section{Platinum-Group Minerals}

Polished thin sections of the three samples with the greatest analyzed PGE values were examined for PGM. The minerals located are $\mathrm{Pt}-, \mathrm{Pd}-$, and Ir-bearing phases. They occur as composite grains up to $10 \mu \mathrm{m}$ in diameter. In most cases, the PGM were of sufficient size to be analyzed quantitatively (Table 5).

The troctolites were found to contain a variety of PGM. In Sample 147-895E-6R-1 (Piece 18), two composite PGM grains were observed. One PGM grain is situated in an irregular intergrowth of NiFe alloys altering to oxides and magnetite on the edge of an intergrowth of pentlandite and magnetite laths (Fig. 10A). The cluster of sulfides, oxides, and PGM is surrounded by silicates now altered to serpentine. This composite PGM grain has an approximately rectangular shape with irregular or rounded edges and is $8 \times 10 \mu \mathrm{m}$ in diameter (Fig. 10B). One edge is formed by a Pd-Sn-Cu alloy $\left(\mathrm{Pd}_{1.02} \mathrm{Sn}_{1,06} \mathrm{Cu}_{1.04}\right)$. The center is composed of a Pt-Fe-Ni-Cu alloy and is separated from the Pd-Sn-Cu alloy by a Ni-Sn alloy. On another edge of the grain lies a cluster of Pt-bearing PGM of Cu-Pt-Au, Pt$\mathrm{Ni}-\mathrm{Cu}$, and $\mathrm{Cu}-\mathrm{Pt}$. The second composite grain is triangular in shape and $4 \mu \mathrm{m}$ in diameter. It is enclosed by a Ni-Fe alloy surrounded on its edges by $\mathrm{Ni}-\mathrm{S}$, which is in a cluster of $\mathrm{Ni}-\mathrm{S}, \mathrm{Ni}-\mathrm{Fe}$ alloys, native copper, and ferritchromit (Fig. 10C). This composite PGM grain consists of a Ni-Pt-Fe alloy containing rounded grains of a very Pt-rich $\mathrm{Ni}-\mathrm{Fe}-\mathrm{Cu}$ alloy (Fig. 10D). Both the PGM grains in this sample occur in alloy and sulfide clusters enclosed by serpentine, which replaces one large (approximately $1 \mathrm{~cm}$ in length) olivine crystal. These large olivines are characteristic of the troctolites.

In a thin section from Sample 147-895D-7R-1 (Piece 11, 71-74 $\mathrm{cm}$ ), an isolated, $1-\mu \mathrm{m}$ grain of a Pt-Cu mineral occurs very close to a pentlandite rimmed by magnetite. Another isolated grain, of Au$\mathrm{Cu}$, also occurs in this section (Fig. 3A).

Troctolite Sample 147-895D-7R-1 (Piece 8, 45-49 cm), contains a composite grain of PGM enclosed in a rounded grain of pentlandite containing exsolution lamellae of chalcopyrite. These, in turn, are enclosed by a chrome-spinel grain surrounded by plagioclase (Fig. $10 \mathrm{E})$. Within the sulfide the composite PGM grain is surrounded by a thin rim of pyrrhotite. The $2.5-\mu \mathrm{m}$ rounded grain of PGM has on its edges a $1 \times 0.5 \mu \mathrm{m}$ mineral of $\mathrm{Pd}-\mathrm{Bi}-\mathrm{Te}$ and a $1 \times 1 \mu \mathrm{m}$ grain of Pt$\mathrm{S}$ (Fig. 3B), and the remainder is composed of a Pd-Pt-Bi-Te mineral of variable composition (Fig. 10F).

The dunite Sample 147-895E-4R-1 (Piece 19, 131-134 cm) contains two adjacent, round, $0.5-\mu \mathrm{m}$ grains of PGM near the edge of, but enclosed by, an isolated $10-\mathrm{mm} \mathrm{Ni}-\mathrm{Fe}$ alloy, which is surrounded by chlorite (Fig. 10G). These PGM are a Pd-Sn alloy and an Ir-Pt alloy with minor $\mathrm{Pd}, \mathrm{Sn}$, and $\mathrm{Cu}$ (Figs. 3C, 10H). The similar composition of other Ni-Fe alloys in the section indicates that there is nothing unusual about the composition of the PGM-bearing Ni-Fe alloy.

\section{DISCUSSION Comparison with Ophiolites}

Traditionally, ancient oceanic crust represented by ophiolite complexes was thought to be enriched only in the PGE Os, Ir, and Ru (Constantinides et al., 1980; Page and Talkington, 1984; Talkington et al., 1984; Stockman and Hlava, 1984; Augé, 1986; Legendre and Augé, 1986). More recently, Pt and Pd have been observed in several ophiolite complexes, generally in association with sulfides and disseminated layers of chromite in thick dunite-rich sequences (Orberger et al., 1988; Ohnenstetter et al., 1991; Leblanc et al., 1991; Pedersen et al., 1993; Prichard and Lord, 1993). The most enriched 

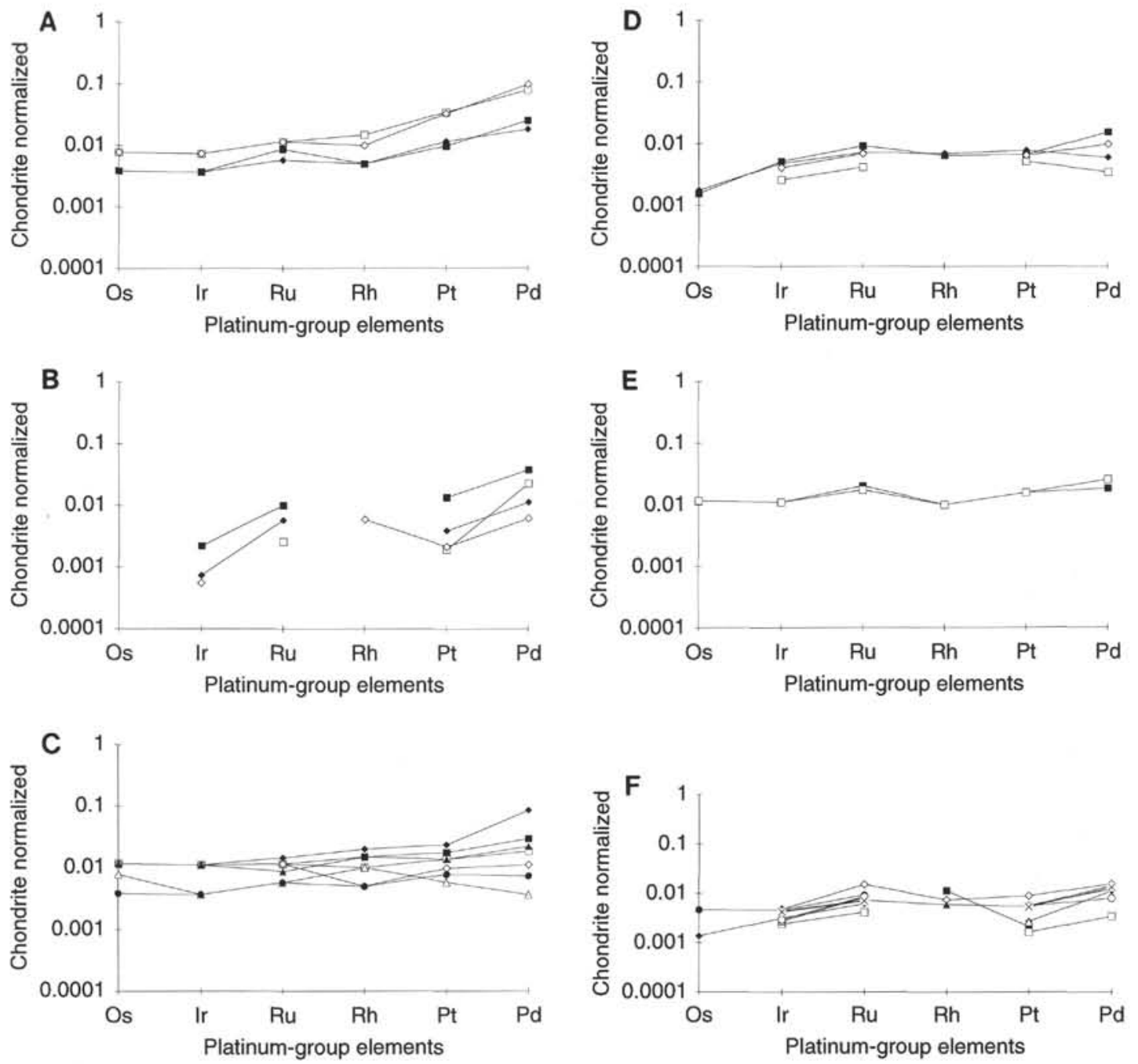

Figure 4. Chondrite-normalized plots for all samples analyzed for PGE at Site 895. Troctolites: (A) data set 1 (Table 1) and (B) data set 2 (Table 2). Dunites: (C) data set 1 (Table 1) and (D) data set 2 (Table 2). Harzburgites: (E) data set 1 (Table 1) and (F) data set 2 (Table 2). Values for chondrite are Os 514 ppb, Ir 540 ppb, Ru 690 ppb, Rh 200 ppb, Pt 1025 ppb, and Pd 545 ppb, taken from Naldrett and Duke (1980).

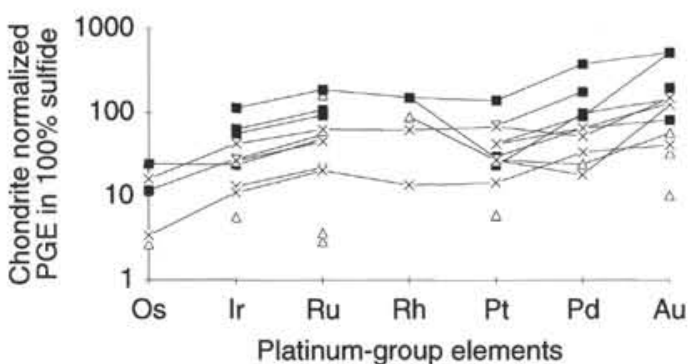

Figure 5. Chondrite-normalized PGE profiles for gabbros from Site 894 (open triangles), dunites (“X”s), and harzburgites (solid squares) from Site 895 .

magmatic PGE values in the ultramafic parts of ophiolite complexes are in the order of 1-2 ppm Pt + Pd (Corrivaux and LaFlamme, 1990; Edwards, 1990; Prichard and Lord; 1993; Ohnenstetter et al., 1991; Pedersen et al., 1993). The PGE concentrations in the Hess Deep ultramafic lithologies also are associated with chrome-spinels and sulfides but are much less enriched. PGE concentrations have been rarely recorded in gabbroic lithologies in ophiolites, but in Oman and
Cyprus $\mathrm{Pt}$ and $\mathrm{Pd}$ occur in gabbros at concentrations similar to those recorded in the troctolites in Hess Deep (i.e., $167 \mathrm{ppb} \mathrm{Pt}+\mathrm{Pd}$ in Oman (Lachize et al., 1991); and $100 \mathrm{ppb}$ in Cyprus (Prichard and Lord, 1990) and these PGE are associated with sulfides in a similar manner to those in the Hess Deep troctolite.

Therefore, although the maximum PGE concentrations in the Hess Deep samples are smaller than those observed in ophiolites, the association of Au minerals, base-metal sulfides, and Cr with PGE-enriched samples in Hess Deep is characteristic also of PGE concentrations in ophiolite complexes (Prichard et al., 1994).

\section{Retention of Platinum-Group Elements in the Mantle}

PGE are thought to be associated with sulfides, and in a magma they are collected by an immiscible sulfide liquid (Naldrett, 1989). Normally, the calculation of PGE in $100 \%$ of sulfide is made for igneous lithologies in which the percentage of sulfide differs from sample to sample so that, assuming that all PGE are collected by the immiscible sulfide, the calculation allows PGE concentrations to be compared for different samples formed at different stages of magma fractionation. For very low concentrations of sulfur, it has been debated whether the PGE reside completely in the sulfides and, therefore, whether a PGE concentration in $100 \%$ of sulfide is justified 

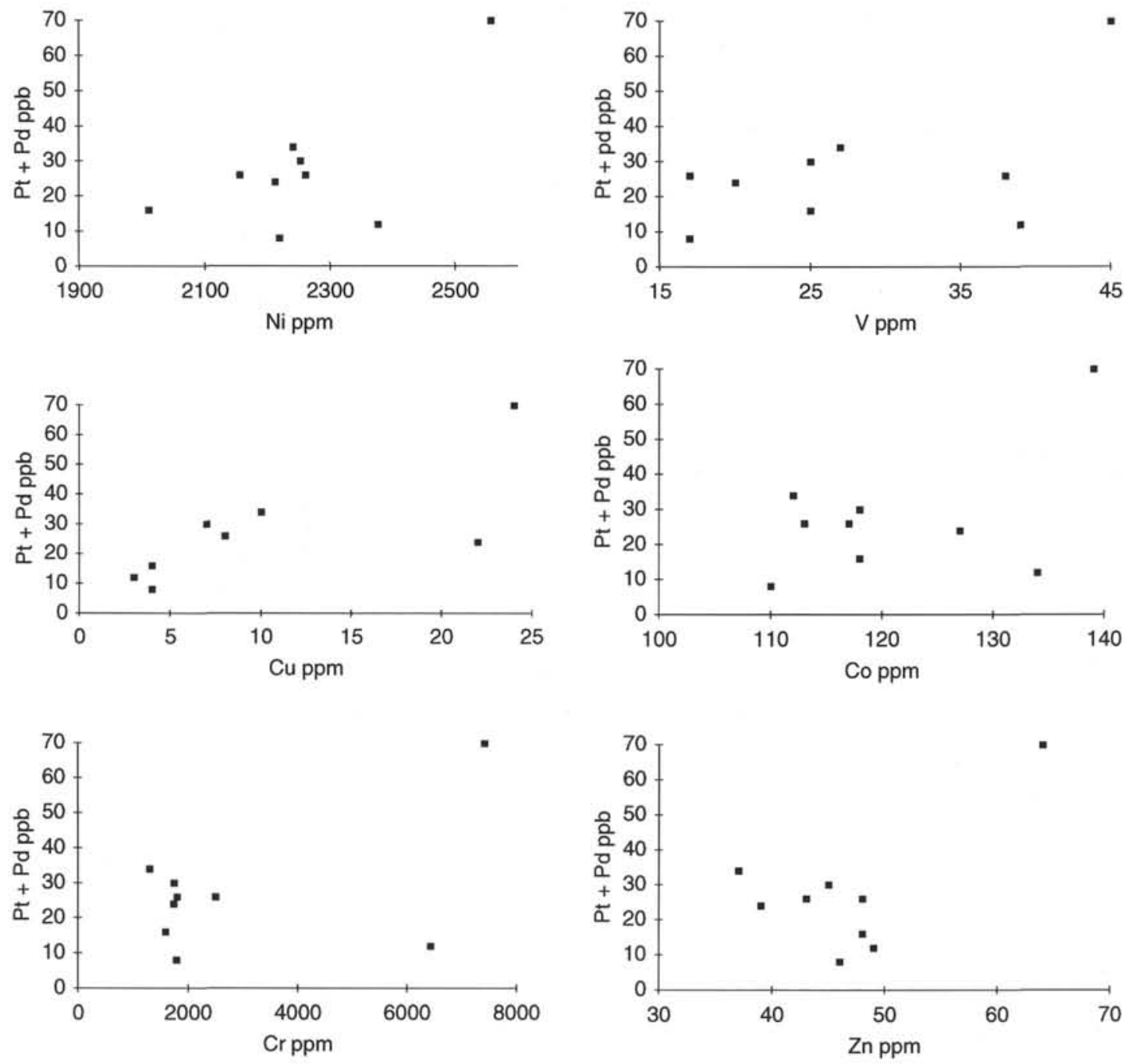

Figure 6. Graphs of Pt + Pd values vs. pathfinder elements for all dunites analyzed for PGE in data set 1 (Table 1). The greatest values of Pt + Pd correspond with the greatest value of each pathfinder element.

(Barnes et al., 1988). However, if it is assumed that all PGE are concentrated with the sulfides in the Hess Deep samples, then it appears that the sulfide fraction of the harzburgite is more enriched in PGE than the sulfide fraction in the dunite or troctolite. Thus, although the harzburgite contains less sulfide than the dunite or troctolite, the sulfide is more PGE rich. This explains the lack of any occurrence of 1to 2-ppm concentrations of PGE in the sulfide- and chrome-spinelbearing dunites from Hess Deep, as some of the PGE has remained in the mantle, resulting in a less PGE-rich magma.

A note of caution is appropriate here, as more anomalous PGE concentrations could occur in lower crustal rocks from Hess Deep. In ophiolite complexes, PGE-enriched lithologies are characterized by discontinuous sulfide-bearing chromite-rich dunites adjacent to chromitite layers. Such layers were not sampled by drilling during Leg 147 but may occur because the drill core is probably not a comprehensive section of all Hess Deep lithologies. PGE-rich lithologies could be present, especially as the lithologies sampled are highly similar to those commonly associated with PGE-enriched lithologies in ophiolites.

Despite this uncertainty, the presence of higher amounts of PGE in $100 \%$ of sulfide in the harzburgite relative to the dunite, and the common presence of sulfides in the harzburgite, compared to ophiolitic harzburgites, is strong evidence that the Hess Deep harzburgite is unusual. This has wider implications for PGE concentrations in different types of oceanic crust (Fig. 11). Theoretically, PGE, which are very refractory, should require high degrees of partial melting for extraction from the mantle. This may occur in subduction zones where water added to the system during subduction causes either greater partial melting or perhaps a second-stage partial melt from an already partially depleted mantle. Many ophiolite complexes are thought to form at supra-subduction zones (SSZ) and so the PGE concentrations in the magmatic ultramafic components of ophiolite complexes should be greater than those observed in Hess Deep, which has not been subjected to subduction. The key piece of evidence from the Hess Deep data that indicates that magmas generated at an SSZ may be rich in PGE is the discovery that the Hess Deep mantle is still fertile in PGE even after the extraction of a mid-oceanic-ridge melt. Thus, there is a source of PGE residing in oceanic mantle that can be released by further melting at a subduction zone.

Little is known about the differences in PGE concentrations between modern-day lower oceanic crust forming at fast- or slowspreading ridges, but greater melting, whether at fast- or slow-spreading ridges, may result in slightly more PGE-enriched sequences, such as at Hess Deep. However, these sequences are not likely to be as enriched as those found above subduction zones, where the degree of partial melting may be even greater. 
Table 6. Pathfinder elements and platinum-group elements for the PGE-rich section of troctolite, Section 147-895D-7R-1 (Pieces 7 through 16).

\begin{tabular}{ccccccrrrrrrrrr}
\hline Piece & $\begin{array}{c}\text { Interval } \\
(\mathrm{cm})\end{array}$ & Lithology & $\mathrm{Pt}$ & $\mathrm{Pd}$ & $\mathrm{Na}_{2} \mathrm{O}$ & $\mathrm{Fe}_{2} \mathrm{O}_{3}$ & $\mathrm{Ni}$ & $\mathrm{Cu}$ & $\mathrm{Cr}$ & $\mathrm{Zn}$ & $\mathrm{Co}$ & $\mathrm{Mn}$ & $\mathrm{S}$ \\
\hline 7 & 41 & $\mathrm{tr}$ & & & 0.10 & 9.0 & 2320 & 259 & 5617 & 55 & 106 & 0.22 & 0.00 \\
8 & 45 & tr & 36 & 44 & 0.07 & 10.1 & 2608 & 324 & 8573 & 62 & 115 & 0.29 & 0.14 \\
10 & 65 & tr & & & 0.06 & 8.7 & 1785 & 86 & 2369 & 43 & 101 & 0.15 & 0.04 \\
12 & 74 & tr & \multirow{2}{*}{12} & 10 & 0.10 & 9.2 & 1926 & 116 & 3404 & 50 & 106 & 0.17 & 0.05 \\
$20 \mathrm{~B}$ & 131 & $\mathrm{hz}$ & & & 0.05 & 9.2 & 2095 & 56 & 3434 & 45 & 105 & 0.20 & 0.03 \\
\hline
\end{tabular}

Notes: $\mathrm{Pt}$ and $\mathrm{Pd}$ in ppb for those samples analyzed. $\mathrm{Ni}, \mathrm{Cu}, \mathrm{Cr}, \mathrm{Zn}$, and $\mathrm{Co}$ are in ppm, and $\mathrm{Mn}, \mathrm{Na}_{2} \mathrm{O}, \mathrm{Fe}_{2} \mathrm{O}_{3}$, and $\mathrm{S}$ are in weight percent. $\mathrm{Lithology}$ : $\mathrm{tr}=$ troctolite, hz $=$ harzburgite.
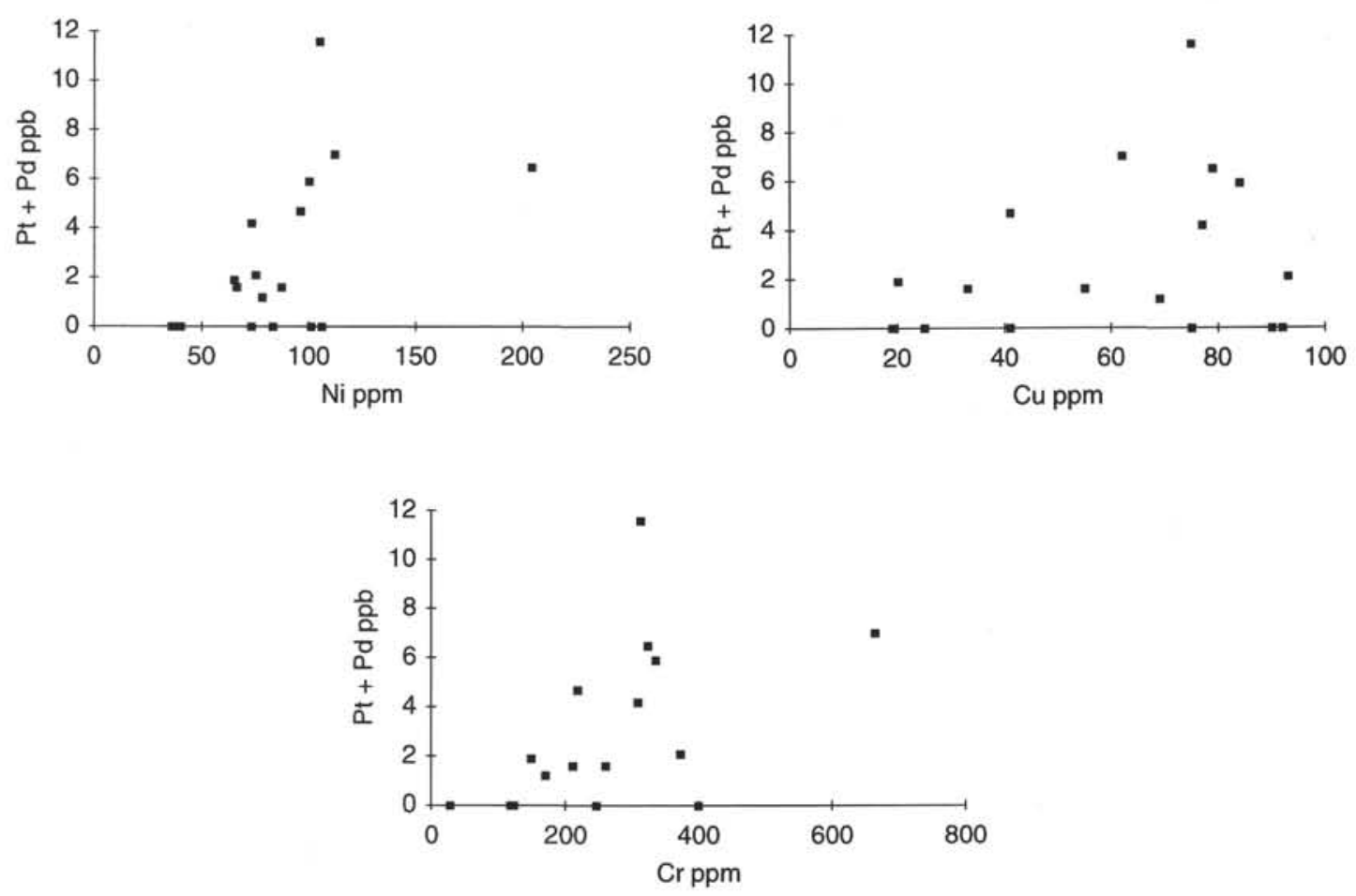

Figure 7. Graphs of $\mathrm{Pt}+\mathrm{Pd}$ values vs. pathfinder elements for the Site 894 gabbros analyzed for PGE (Table 2).

\section{Fractionation of the Platinum-Group Elements}

The troctolite Section 147-895D-7R-1 (Pieces 7 through 16) appears to show an increase of $\mathrm{Ni}, \mathrm{Cu}, \mathrm{Mn}, \mathrm{Zn}, \mathrm{Cr}$, and PGE (expressed mineralogically as sulfides, chrome-spinel, and PGM) toward the top of the section. Inspection of the core reveals also that the troctolites become richer in mafic materials upward. The origin of the troctolite in the Hess Deep cores is controversial. Dick (this volume) argued that the mineralogy of the troctolite has formed or been moderated by in situ impregnation from the adjacent ultramafic rocks. He observed that the plagioclases in the troctolite have reversed zoning with sodic cores and Ca-rich rims. The plagioclase in this troctolite Section $147-$ 895D-7R-1 (Pieces 7 through 16) is too altered for zoning to be preserved. An alternative is that the troctolite formed by crystallization from a fractionated magma, which would be supported by fractionation trends. Impregnation might be expected to produce symmetrical characteristics across troctolite sections whereas fractionation would produce asymmetrical trends. This study neither supports the impregnation nor the fractionation hypothesis but may demonstrate a reverse fractionation trend.

Fractionation of the PGE is more clearly demonstrated by the variation in PGM composition in the dunites and troctolites. The distribution of individual PGE usually depends on the degree of fractionation. $\mathrm{Os}, \mathrm{Ir}$, and $\mathrm{Ru}$ have higher melting points than $\mathrm{Pt}$ and $\mathrm{Pd}$ and are concentrated in more primitive igneous lithologies (Barnes et al., 1988). The only Ir-bearing PGM from Hess Deep was located in the dunite rather than the more evolved troctolite, which contains only Pt- and Pd-bearing PGM.

\section{Platinum-Group Elements in Gabbros}

Samples are rarely analyzed for both REE and PGE, as the PGE tend to be concentrated in more ultramafic lithologies and REE in more fractionated lithologies. This unusual data set of both analyses results from a combination of low analytical detection limits and the degree of fractionation of the Site 894 gabbros, in which both PGE and REE are slightly concentrated because the PGE are not quite depleted and the REE are just becoming enriched. The correlation of gabbros slightly enriched in Pt $+\mathrm{Pd}$ with HREE- and especially LREE-depleted patterns and the lack of a Eu anomaly suggests that these slightly $(\mathrm{Pt}+\mathrm{Pd})$-enriched gabbros are less fractionated than the $(\mathrm{Pt}+\mathrm{Pd})$-poor gabbros and have not undergone plagioclase fractionation. This is consistent with the concentration of $\mathrm{Pt}+\mathrm{Pd}$ in more primitive gabbros, as PGE are highly incompatible in magmas and consequently crystallize early.

\section{Formation of the Platinum-Group Minerals}

The association of PGE with base-metal sulfides indicates that the PGE were probably collected by an immiscible sulfide magma, a pro- 
Table 7. REE analyses (ppm) for gabbros that were also analyzed for platinum-group elements (Table 2).

\begin{tabular}{|c|c|c|c|c|c|c|c|c|c|c|c|c|c|}
\hline $\begin{array}{l}\text { Core, section, } \\
\text { interval }(\mathrm{cm})\end{array}$ & $\begin{array}{l}\text { Depth } \\
\text { (mbsf) }\end{array}$ & Lithology & La & $\mathrm{Ce}$ & $\mathrm{Nd}$ & $\mathrm{Sm}$ & Eu & Gd & $\mathrm{Tb}$ & Ho & $\operatorname{Tm}$ & $\mathrm{Yb}$ & Lu \\
\hline \multicolumn{14}{|l|}{$147-894 \mathrm{~F}$} \\
\hline 3R-1 (Piece 17, 98-103) & 17.79 & $\mathrm{~g}$ & 3.2 & 3.2 & 4.1 & 5.6 & 7.3 & & 7.0 & 7.2 & & 6.0 & 5.6 \\
\hline \multicolumn{14}{|l|}{ 147-894G- } \\
\hline 2R-2 (Piece 15,95-100) & 31.05 & BS & 4.5 & 5.7 & 7.8 & 10.2 & 10.9 & 9.8 & 11.2 & 10.9 & 10.2 & 10.1 & 9.3 \\
\hline 2R-3 (Piece 14, 120-122) & 32.75 & ol g & 2.3 & 5.0 & 4.7 & 5.0 & 7.9 & & 8.2 & & 6.5 & 7.0 & 6.5 \\
\hline 6R-1 (Piece 8A, 81-85) & 55.61 & $\mathrm{gn}^{\circ}$ & 7.4 & 9.1 & 13.6 & & 12.9 & 11.7 & 16.0 & & 11.1 & 14.0 & 11.8 \\
\hline 6R-2 (Piece 1, 79-87) & 56.35 & gn & 13.4 & 16.3 & 19.6 & 19.2 & 15.6 & 26.9 & 19.8 & & 13.0 & 18.2 & 15.5 \\
\hline 6R-2 (Piece 6, 79-87) & 57.02 & gn & 3.8 & 5.9 & 6.2 & 7.9 & 9.1 & 11.6 & 10.3 & 10.3 & 9.3 & 8.4 & 7.1 \\
\hline 6R-2 (Piece 10, 113-117) & 57.36 & gn & 3.9 & 5.4 & 5.6 & 8.9 & 10.6 & & 11.2 & & 7.1 & 10.4 & 9.6 \\
\hline 6R-2 (Piece 14, 141-143) & 57.64 & gn & 4.7 & 6.2 & 9.3 & 8.9 & 10.2 & & 10.3 & 7.0 & 7.1 & 9.6 & 9.0 \\
\hline 9R-2 (Piece 1C, 28-29) & 75.93 & gn & 4.8 & 8.1 & 9.4 & 11.6 & 13.6 & 17.1 & 14.1 & 7.0 & 8.6 & 12.1 & 9.6 \\
\hline 9R-2 (Piece 5F, 115-120) & 76.80 & gn & 12.5 & 15.2 & 14.9 & 16.3 & 17.3 & 21.0 & 17.9 & 17.7 & 12.7 & 15.6 & 13.0 \\
\hline $11 \mathrm{R}-3$ (Piece $3,17-28$ ) & 87.14 & gn & 9.2 & 12.3 & 15.0 & 16.8 & 14.1 & 17.1 & 20.9 & 18.1 & 17.0 & 17.1 & 14.9 \\
\hline 13R-1 (Piece 7B, 42-48) & 103.9 & gn & 3.5 & 7.0 & 7.7 & 9.8 & 11.7 & 7.1 & 13.9 & 12.0 & 10.8 & 10.2 & 9.9 \\
\hline 13R-2 (Piece 9A, 100-107) & 105.8 & gn & 1.9 & 3.9 & 4.1 & 6.6 & 9.5 & 9.2 & 9.7 & 7.7 & 6.2 & 7.9 & 6.2 \\
\hline 13R-2 (Piece 10C, 132-137) & 106.1 & gn & 4.4 & 7.0 & 8.5 & 10.0 & 10.2 & 11.4 & 12.4 & 8.8 & 8.3 & 11.6 & 9.6 \\
\hline 13R-3 (Piece 1C, 17-24) & 106.4 & gn & 1.2 & 2.7 . & 3.1 & 5.1 & 7.6 & 6.6 & 8.4 & 7.5 & & 6.4 & 5.3 \\
\hline 17R-1 (Piece 12, 95-99) & 126.8 & gn & 2.2 & 4.4 & 2.2 & 6.5 & 8.8 & & 7.8 & & 8.0 & 8.3 & 7.5 \\
\hline
\end{tabular}

Notes: $\mathrm{g}=$ gabbro, $\mathrm{BS}=$ basalt, ol $\mathrm{g}=$ olivine gabbro, $\mathrm{gn}=$ gabbronorite. Blank $=$ not determined.
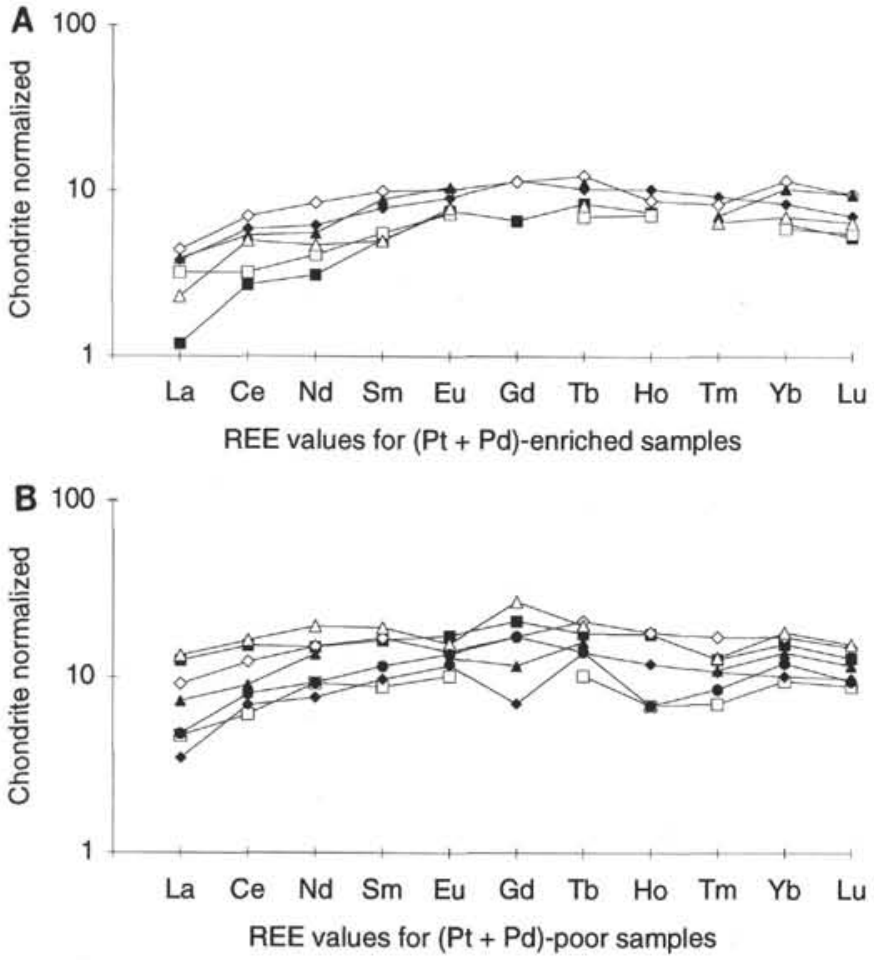

Figure 8. REE patterns for gabbros from Site 894 from data set 2 (Table 2). Boynton-chondrite values used are $\mathrm{La} 0.31, \mathrm{Ce} 0.808, \mathrm{Nd} \mathrm{0.6,} \mathrm{Sm} \mathrm{0.195,} \mathrm{Eu}$ 0.0735 , Gd 0.259, Tb 0.0474, Ho 0.0718, Tm 0.0324, Yb 0.209, and Lu 0.0322 . A. Analyses $1,3,6,7,14$, and 15 in Table 7. B. Analyses 4, 5, 8-13, and 16 in Table 7.

cess that has been suggested for the origin of PGE concentrations at Noril'sk (Genkin and Evstigneeva 1986; Czamanske et al., 1992) and Sudbury (Li et al., 1992) and in ophiolites (Prichard et al., 1994). Whether the PGM (1) crystallized in solid solution with the sulfides as they were precipitated from the magma and subsequently were released from the sulfides to form discrete PGM during alteration of the sulfides perhaps during serpentinization or (2) crystallized directly as discrete PGM adjacent to the sulfides is uncertain and controversial. The arguments are summarized in Prichard et al. (1994). In the Hess Deep samples, as elsewhere, it is difficult to determine which hypoth-

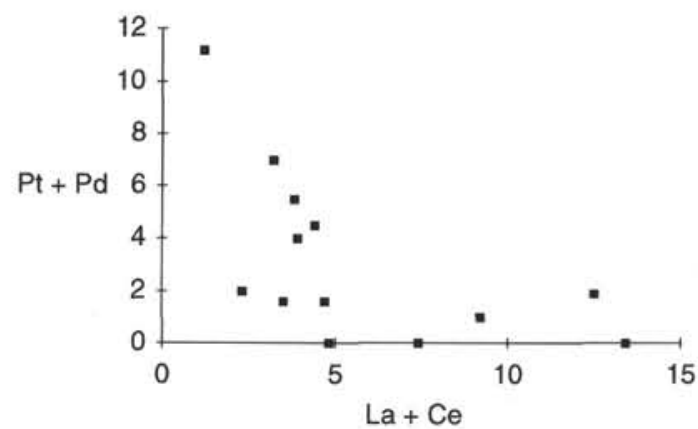

Figure 9. Graph of Pt $+\mathrm{Pd}(\mathrm{ppb})$ vs. the light REE (ppm) $\mathrm{La}+\mathrm{Ce}$

esis is correct because the samples are altered. Although pentlandite and chalcopyrite are present, the dominant mineral assemblage is almost totally secondary, probably having formed during serpentinization (Dick, 1974; Ramdohr, 1967). Thus, base metals are present as alloys such as Ni-Fe and pentlandite is commonly altered to $\mathrm{Ni}$ sulfides and magnetite. Native copper is common. Most of the PGM are also alloys and so are likely to represent secondary phases formed at the same time as the base-metal alloys. The close association of PGM with base-metal sulfides not only implies a magmatic origin but also necessitates alteration in situ, without significant mobilization of the PGE during the mineralogical transformation; thus the magmatic chondrite-normalized profiles are preserved. Of additional importance for the timing of serpentinization and alteration of the PGE mineralogy in ophiolite complexes is the fact that the Hess Deep samples have the alteration assemblage commonly observed in ophiolite complexes. This suggests that alteration can occur in the oceanic crust very soon $(<1 \mathrm{Ma})$ after its formation and prior to ophiolite obduction and emplacement.

\section{CONCLUSIONS}

Despite the fact that the length of drill core obtained during Leg 147 was less than on most sedimentary legs, the variety of lithologies in continuous core section made possible the location of PGE concentrations and enabled specific PGE associations with lithologic sequences to be observed in a way that would have been impossible using dredged samples. The data show conclusively that PGE concentrations occur in fast-spreading unsubducted oceanic crust and that the mantle harzburgite remains partially fertile in PGE. The pres- 
ence and distribution between mantle and lower oceanic crust of the PGE concentrations at Hess Deep have major implications for the distribution of PGE in the Earth's oceanic crust and for the global PGE cycle. Although the PGE concentrations described are small, there are vast areas of oceanic crust and mantle produced and modified by mid-oceanic-ridge processes similar to those that formed the Hess Deep lithologies. Further research on the distribution of PGE in oceanic crust generated at different spreading centers, perhaps with different spreading rates, is necessary to determine how and to what extent the highly refractory chalcophile elements, such as the PGE, are extracted from the mantle and concentrated in the lower oceanic crust during varying degrees of partial melting.

\section{ACKNOWLEDGMENTS}

We are grateful to the international ODP organization for allowing us to participate in this research and to NERC and the Royal Society for funding. We would like to thank all the scientists on board JOIDES Resolution during Leg 147 for interesting discussions that helped us produce this paper. We would like to thank Terry Wheeler of Genalysis for providing the PGE analyses for data set 1 .

\section{REFERENCES}

Agiorgitis, G., and Wolf, R., 1978. Aspects of osmium, ruthenium and iridium contents in some Greek chromites. Chem. Geol., 23:267-272.

Augé, T., 1986. Platinum-group mineral inclusions in ophiolitic chromitites from the Oman ophiolite. Bull. Mineral., 109:301-304.

Barnes, S.J., Boyd, R., Korneliussen, A., Nilsson, L.-P., Often, M., Pedersen, R.B., and Robins, B., 1988. The use of mantle normalization and metal ratios in discriminating between the effects of partial melting, crystal fractionation and sulphide segregation on platinum-group elements, gold, nickel and copper: examples from Norway. In Prichard, H.M., Potts, P.J., Bowles, J.F.W., and Cribb, S.J. (Eds.), Geo-Platinum '87: Essex (Elsevier), 113-144.

Constantinides, C.C., Kingston, G.A., and Fisher, P.C., 1980. The occurrence of platinum-group minerals in the chromitites of the Kokkinorotsos chrome mine, Cyprus. In Panayiotou, A. (Ed.), Ophiolites: Proc. Int. Ophiolite Symp. Cyprus, Geol. Surv. Dept. Nicosia, 93-101.

Corrivaux, L., and LaFlamme, J.H.G., 1990. Minerlogie des elements du groupe du platine dans les chromitites de l'ophiolite de Thetford mines, Quebec. Can. Mineral., 28:579-595.

Czamanske, G.K., Kunilov, V.E., Zientek, M.L., Cabri, L.J., Likkhachev, A.P., Calk, L.C., and Oscarson, R.L., 1992. A proton-microprobe study of magmatic sulfide ores from the Noril'sk-Talnakh district, Siberia. Can. Mineral., 30:249-287.

Dick, H.J.B., 1974. Terrestrial nickel-iron from the Josephine peridotite, its geologic occurrence, associations and origin. Earth Planet. Sci. Lett., 24:291-298.

Edwards, S.J., 1990. Harzburgites and refractory melts in the Lewis Hills massif, Bay of Islands ophiolite complex: the base metals and precious metals story. Can. Mineral., 28:537-552.

Genkin, A.D., and Evstigneeva, T.L., 1986. Associations of platinum-group minerals of the Noril'sk copper-nickel sulfide ores. Econ. Geol., $81: 1203-1212$.

Greenbaum, D., 1977. Magmatic processes at ocean ridges, evidence from the Troodos massif, Cyprus. Nature, 288:18-21.

Lachize, M., Lorand, J.P., and Juteau, T., 1991. Cu-Ni-PGE magmatic sulfide ores and their host layered gabbros in the Haymiliyah fossil magma chamber (Haylayn block, Semail Ophiolite nappe, Oman). In Peters, T., Nicolas, A., and Coleman, R.J. (Eds.), Ophiolite Genesis and Evolution of the Oceanic Lithosphere. (Kluwer) 5:209-229.

Leblanc, M., Ceuleneer, G., Al Azri, H., and Jedwab, J., 1991. Concentration hydrothermale de palladium et de platine dans les péridotites mantellaires du complexe ophiolitique d'Oman. C. R. Acad. Sci. Ser. 2, 312:10071012.

Legendre, O., and Augé, T., 1986. Mineralogy of platinum-group mineral inclusions in chromitites from different ophiolitic complexes. In Gallagher, M.J., Ixer, R.A., Neary, C.R., and Prichard, H.M. (Eds.), Metallogeny of Basic and Ultrabasic Rocks: London (Inst. Min. Metall.), 361375.
Li, C., Naldrett, A.J., Coats, C.J.A., and Johannessen, P., 1992. Platinum, palladium, gold, and copper-rich stringers at the Strathcona mine, Sudbury: their enrichment by fractionation of a sulfide liquid. Econ. Geol., 87:1584-1598.

Naldrett, A.J., 1989. Magmatic Sulphide Deposits. Oxford Monogr. Geol. Geophys., 14.

Naldrett, A.J., and Duke, J.M., 1980. Pt metals in magmatic sulfide ores: the occurrence of these metals is discussed in relation to the formation and importance of these ores. Science, 208:1417-1424.

Neary, C.R., and Brown, M.A., 1979. Chromites from the Al Ays complex, Saudi Arabia and the Semail complex, Oman. In Al Shanti, A.M.S. (Ed.), Evolution and Mineralisation of the Arabian Shield. Inst. Arabian Geologists Bull., 2: 193-205.

Ohnenstetter, M., Karaj, N., Neziraj, A., Johan, Z., and Cina, A., 1991. Le potentiel platinifere des ophiolites: minéralisations en éléments du groupe du platine (PGE) dans les massifs de Tropoja et Bulqiza, Albanie. C. R. Acad. Sci. Ser. 2, 313:201-208.

Orberger, B., Fredrich, G., and Woermann, E., 1988. Platinum-group element mineralisation in the ultramafic sequence of the Acoje ophiolite block, Zambales, Philippines. In Prichard, H.M., Potts, P.J., Bowles, J.F.W., and Cribb, S.J. (Eds.), Geo-Platinum '87: Essex (Elsevier), 361380.

Page, N.J., Cassard, D., and Haffety, J., 1982. Palladium, platinum, rhodium, ruthenium, and iridium in chromitites from the Massif du Sud and Tiebaghi Massif, New Caledonia. Econ. Geol., 77:1571-1577.

Page, N.J., Engin, T., Singer, D.A., and Haffety, J., 1984. Distribution of platinum-group elements in the Bati Kef chromite deposit, GulemanElazig area, eastern Turkey. Econ. Geol., 79:177-184.

Page, N.J., and Talkington, R.W., 1984. Palladium, platinum, rhodium, ruthenium, and iridium in peridotites and chromites from ophiolite complexes in Newfoundland. Can. Mineral., 22:137-149.

Pedersen, R.B., Johannesen, G.M., and Boyd, R., 1993. Stratiform PGE mineralisations in the ultramafic cumulates of the Leka ophiolite complex, central Norway. Econ. Geol., 88:782-803.

Prichard, H.M., Ixer, R.A., Lord, R.A., Maynard, J., and Williams, N., 1994. Platinum-group mineral assemblages in silicate lithologies and chromiterich rocks within the Shetland ophiolite sequence. Can. Mineral., 32:271-294.

Prichard, H.M., and Lord, R.A., 1988. The Shetland ophiolite: evidence for a supra-subduction zone origin and implications for platinum-group element mineralization. In Prichard, H.M., Potts, P.J., Bowles, J.F.W., and Cribb, S.J. (Eds.), Geo-Platinum '87: Essex (Elsevier), 161.

1990. Platinum and palladium in the Troodos ophiolite complex, Cyprus. Can. Mineral., 28:607-617.

, 1993. An overview of the PGE concentrations in the Shetland ophiolite complex. In Prichard, H.M., Alabaster, T., Harris, N.B.W., and Neary, C.R. (Eds.), Magmatic Processes and Plate Tectonics. Geol. Soc. Spec. Publ. London, 76:273-294.

Prichard, H.M., Neary, C.R., and Potts, P.J., 1986. Platinum-group minerals in the Shetland Ophiolite. In Gallagher, M.J., Ixer, R.A., Neary, C.R., and Prichard, H.M. (Eds.), Metallogeny of Basic and Ultrabasic Rocks: London (Inst. Min. Metall.), 395-414.

Ramdohr, P., 1967. A widespread mineral association connected with serpentinization. Neues Jahrb. Mineral. Abh., 107:241-265.

Roberts, S., and Neary, C.R., 1993. Petrogenesis of ophiolitic chromite. In Prichard, H.M., Alabaster, T., Harris, N.B.W., and Neary, C.R. (Eds.), Magmatic Processes and Plate Tectonics. Geol. Soc. Spec. Publ. London, 76:257-272.

Stockman, H.W., and Hlava, P.F., 1984. Platinum-group minerals in Alpine chromitites from southwestern Oregon. Econ. Geol., 79:491-508.

Talkington, R.W., Watkinson, D.H., Whittaker, P.J., and Jones, P.C., 1984. Platinum group minerals and other solid inclusions in chromites of ophiolitic complexes: occurrence and petrological significance. TMPM, Tschermaks Mineral. Petrogr. Mitt., 32:285-300.

Von Gruenewaldt, G., Hatton, C.J., Merkle, R.K.W., and Gain, S., 1986. PGE-chromite associations in the Bushveld complex. Econ. Geol., 81:1067-1079.

Date of initial receipt: 2 August 1994

Date of acceptance: 2 February 1995

Ms 147S-004 

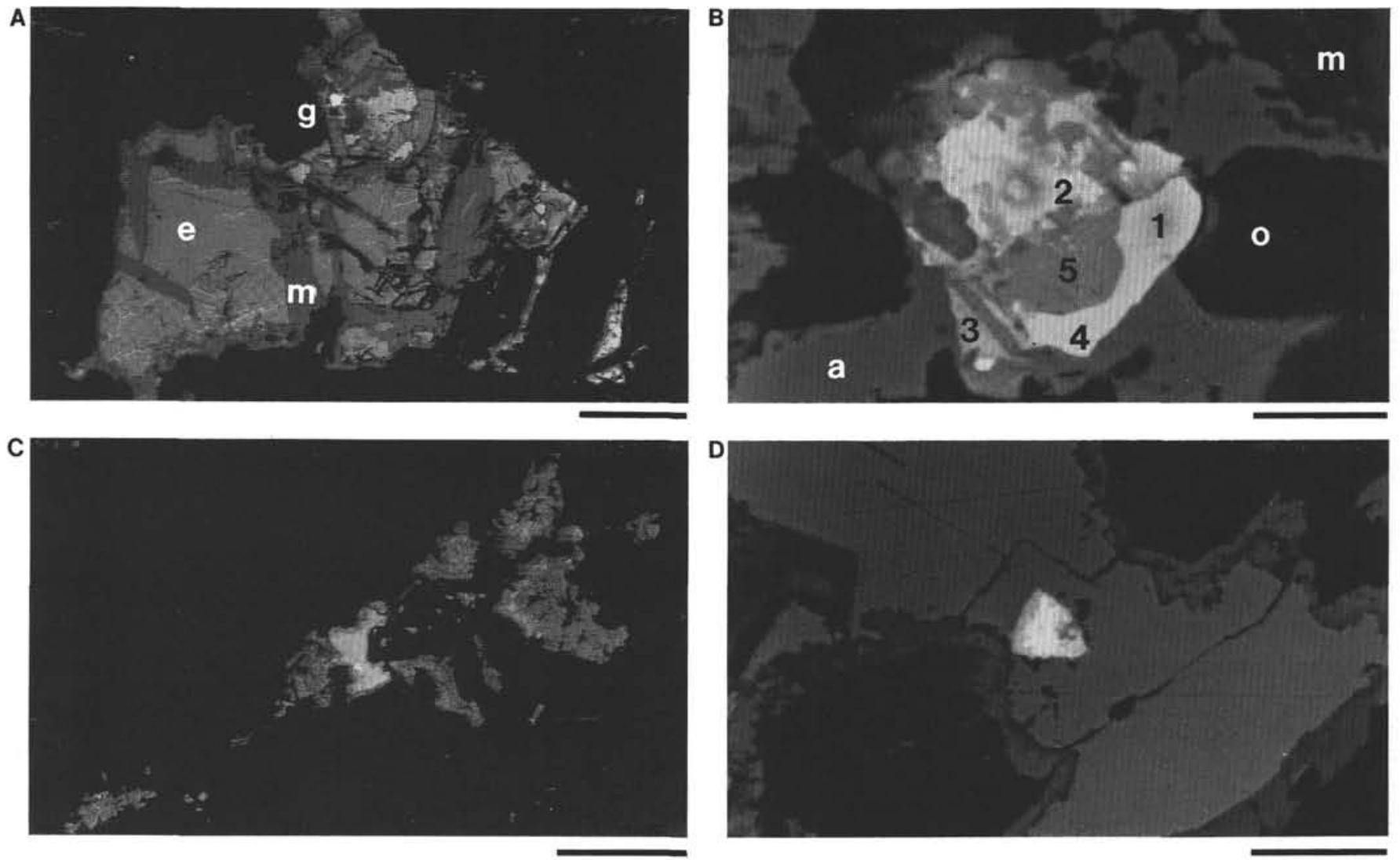

Figure 10. Photomicrographs of PGM. A. Composite PGM grain from Sample 147-895E-6R-1 (Piece 17, 90-92 CM). The PGM (g) appear white in a pale gray pentlandite (e) intergrown with darker gray magnetite laths $(\mathrm{m})$ and surrounded by silicates (black). Scale bar $=100 \mu \mathrm{m}$. B. Close-up of Figure 10A. The composite PGM grain $(10 \times 10 \mu \mathrm{m})$ is surrounded by Ni-Fe alloys (a) altering to oxides (o) and magnetite. The PGM are (1) Pd-Sn-Cu alloy, (2) Pt-Fe-Ni-Cu alloy, (3) Cu-Pt-Au alloy, and (4) Pt-Ni-Cu alloy; (5) is an Ni-Sn alloy. The numbers correspond to analyses in Table 5. Scale bar $=5 \mu \mathrm{m}$. C. Composite PGM grain from Sample 147-895E-6R-1 (Piece 18) appears white enclosed by a Ni-Fe alloy (pale gray) and in a row of grains of ferritchromit (dark gray), all surrounded by silicates (black). Scale bar $=100 \mu \mathrm{m}$. D. Close-up of Figure 10C. The white composite PGM grain consists of a very Pt-rich Ni-Fe-Cu alloy (analyses 6 and 7, Table 5) surrounded by a pale gray Ni-Pt-Fe alloy (analysis 8 , Table 5) and triangular-shaped $(2.5 \times 2.5 \times 2.5 \mu \mathrm{m}) \mathrm{PGM}$ enclosed in a gray Ni-Fe alloy altered on the edges to Ni-S (dark gray), which is in turn surrounded by silicates (black). The numbers correspond to analyses in Table 5 . Scale bar $=10 \mu \mathrm{m}$. E. Composite grain in Sample 147-895D-7R-1 (Piece 8, 45-49 cm) of PGM appears white enclosed in a pale gray rounded grain of pentlandite (e) surrounded by gray chromite, which is surrounded by plagioclase (black). Scale bar $=100 \mu \mathrm{m}$. F. Close-up of Figure 10E. The 4- $\mu \mathrm{m}$ PGM (white) contains Pd-Bi-Te (left) and Pt-S (right) on the edges of a very pale gray Pd-Pt-Bi-Te grain (analysis 9, Table 5). The PGM grain is surrounded by gray pentlandite containing dark gray exsolution lamellae of chalcopyrite. Adjacent to the PGM grain is an irregular halo of pyrrhotite (dark gray). Scale bar $=10 \mu \mathrm{m}$. G. Serpentinized dunite with stringers of magnetite (white) in Sample 147-895E-4R-1 (Piece 19, 131-134 cm) contains an isolated Ni-Fe alloy (a) containing PGM. Scale bar $=50 \mu \mathrm{m}$. H. Close-up of Figure 10G. Two adjacent, round, approximately 1- $\mu \mathrm{m}$ PGM grains (white) near the edge of the Ni-Fe alloy (a) surrounded by fibrous chlorite (dark gray). PGM are an Ir-Pt alloy $(r)$ and a Pd-Sn alloy (s). Scale bar $=5 \mu \mathrm{m}$. 

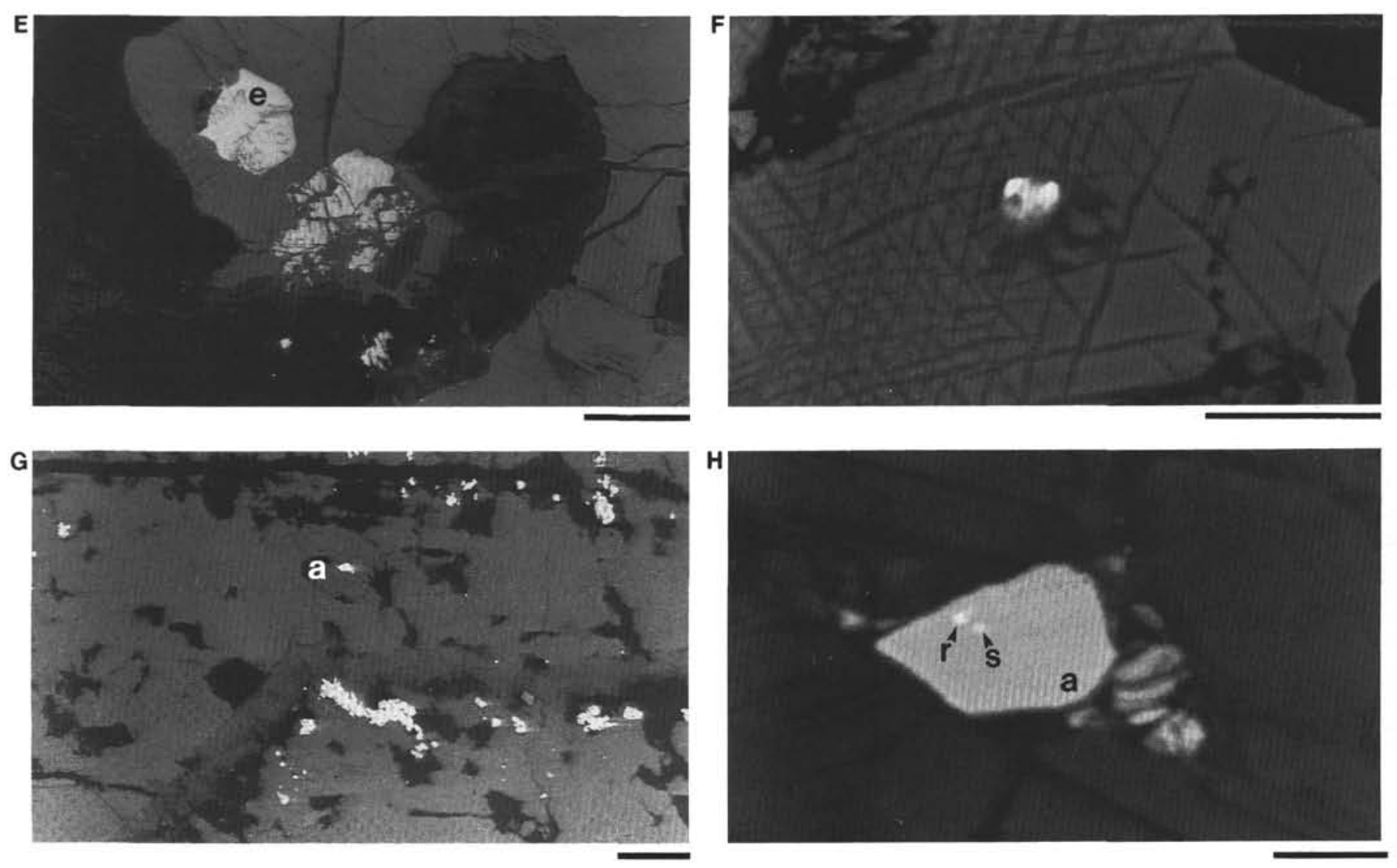

Figure 10 (continued).

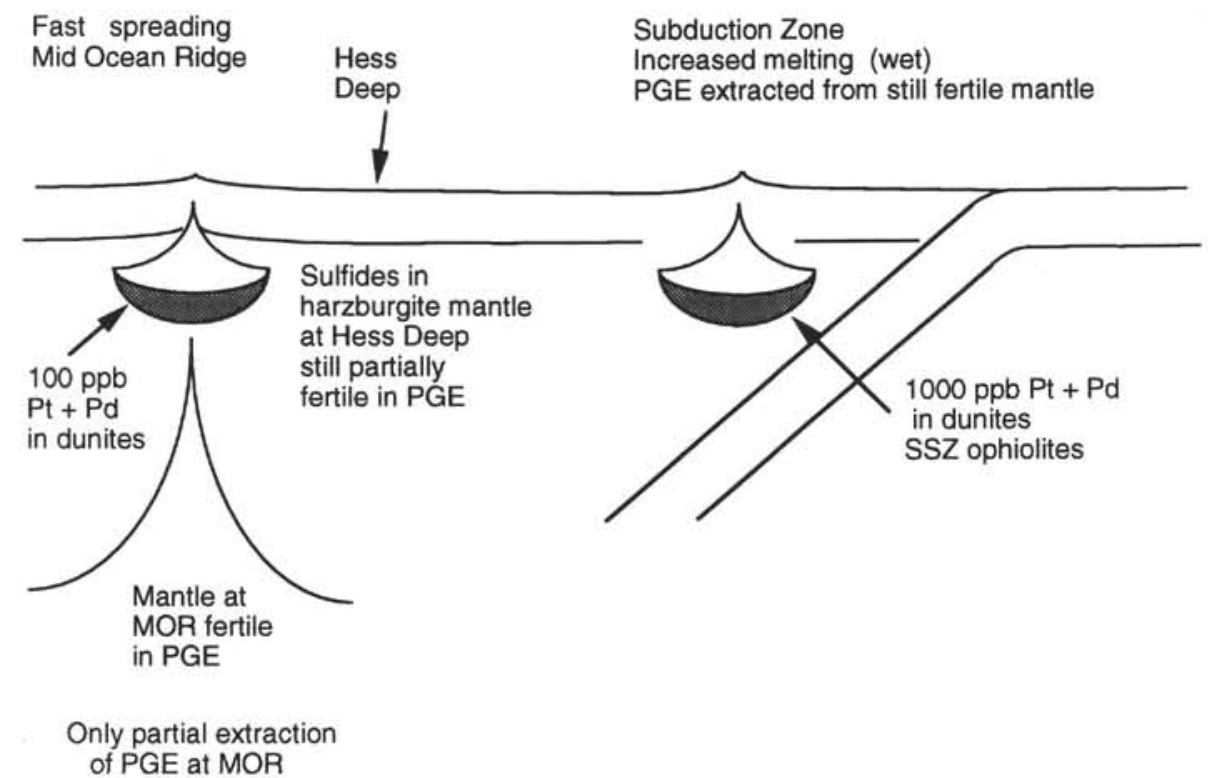

Figure 11. Schematic representation of PGE concentrations in cumulates related to mantle depletion in mid-oceanic ridge (MOR) and supra-subduction zone settings. 